\title{
Quantitative functional renormalization group description of the two-dimensional Hubbard model
}

\author{
Cornelia Hille $\odot,{ }^{1}$ Fabian B. Kugler $\odot,{ }^{2}$ Christian J. Eckhardt, ${ }^{3,4,5}$ Yuan-Yao He,${ }^{6,7}$ Anna Kauch $\odot,{ }^{3}$ Carsten Honerkamp, ${ }^{4,5}$ \\ Alessandro Toschi $\odot,{ }^{3}$ and Sabine Andergassen $\odot^{1}$ \\ ${ }^{1}$ Institut für Theoretische Physik and Center for Quantum Science, Universität Tübingen, Auf der Morgenstelle 14, 72076 Tübingen, Germany \\ ${ }^{2}$ Arnold Sommerfeld Center for Theoretical Physics, Center for NanoScience, and Munich Center for Quantum Science and \\ Technology, Ludwig-Maximilians-Universität München, 80333 Munich, Germany \\ ${ }^{3}$ Institute of Solid State Physics, Vienna University of Technology, 1040 Vienna, Austria \\ ${ }^{4}$ Institute for Theoretical Solid State Physics, RWTH Aachen University, 52056 Aachen, Germany \\ ${ }^{5}$ JARA-FIT, JARA-HPC, Jülich Aachen Research Alliance, 52425 Jülich, Germany \\ ${ }^{6}$ Center for Computational Quantum Physics, Flatiron Institute, New York, New York 10010, USA \\ ${ }^{7}$ Department of Physics, College of William and Mary, Williamsburg, Virginia 23187, USA
}

(Received 10 February 2020; revised 11 August 2020; accepted 12 August 2020; published 8 September 2020)

\begin{abstract}
Using a leading algorithmic implementation of the functional renormalization group (fRG) for interacting fermions on two-dimensional lattices, we provide a detailed analysis of its quantitative reliability for the Hubbard model. In particular, we show that the recently introduced multiloop extension of the fRG flow equations for the self-energy and two-particle vertex allows for a precise match with the parquet approximation also for twodimensional lattice problems. The refinement with respect to previous fRG-based computation schemes relies on an accurate treatment of the frequency and momentum dependences of the two-particle vertex, which combines a proper inclusion of the high-frequency asymptotics with the so-called truncated unity fRG for the momentum dependence. The adoption of the latter scheme requires, as an essential step, a consistent modification of the flow equation of the self-energy. We quantitatively compare our fRG results for the self-energy and momentumdependent susceptibilities and the corresponding solution of the parquet approximation to determinant quantum Monte Carlo data, demonstrating that the fRG is remarkably accurate up to moderate interaction strengths. The presented methodological improvements illustrate how fRG flows can be brought to a quantitative level for two-dimensional problems, providing a solid basis for the application to more general systems.
\end{abstract}

DOI: 10.1103/PhysRevResearch.2.033372

\section{INTRODUCTION}

Renormalization group ( $R G$ ) methods have a long history in theoretical physics, ranging from a way to treat divergences in quantum field theories [1], critical phenomena [2,3], and quantum impurity problems [4,5] to current attempts to elucidate deep learning algorithms by physics [6]. In general, RG methods connect specific quantities of a theory, such as coupling constants or correlation functions, at a given scale with those at another scale via differential equations. This leads to a flow of these quantities which under appropriate circumstances distills out the dominating, and to some degree universal, properties of the system.

The origins of the RG for electron lattice models date back to the second decade of the cuprate high-temperature superconductors more than 20 years ago [7-11]. Here the RG was utilized as a tool to deal with competing ordering tendencies in the Hubbard model (which are also seen in

Published by the American Physical Society under the terms of the Creative Commons Attribution 4.0 International license. Further distribution of this work must maintain attribution to the author(s) and the published article's title, journal citation, and DOI. the cuprates) and as a method to understand in principle the stability of the Landau-Fermi-liquid state [12]. While the aptness of RG schemes in competing-order situations had already been known from impurity [4] and one-dimensional models [13], the systematic and versatile functional RG schemes [12,14-16] turned out to be advantageous in the study of two-dimensional (2D) lattice models such as the Hubbard model, beyond more general considerations [17,18].

The name functional RG (fRG) can be understood as having a twofold reason. On the one hand, the $\mathrm{RG}$ flow is derived from an exact flow equation for a generating functional of the theory when a (suitably chosen) parameter $\Lambda$ in the free quadratic part of the action is changed. On the other hand, one usually investigates the flow of continuous functions of variables $k$ such as wave vectors and frequencies with $\Lambda$, i.e., one deals with differential equations in $\Lambda$ for functions $f_{\Lambda}(k)=f(\Lambda, k)$ of $k$ and $\Lambda$. This marks a difference with respect to the conventional $\mathrm{RG}$, where only a small finite number of constants are flowing. The main objects of interest in the fRG flows we discuss here are the electron singleparticle self-energy $\Sigma_{\Lambda}\left(k_{1}, k_{2}\right)$ and the two-particle (interaction) vertex $V_{\Lambda}\left(k_{1}, k_{2}, k_{3}, k_{4}\right)$. While the self-energy determines the changes of the single-particle excitations due to the mutual interactions, the two-particle correlation functions and collective properties are mostly controlled by the two-particle 
scattering processes, the so-called vertex correction terms. A dominant role played by the latter clearly emerged from model [19-27] and realistic [28-32] calculations of correlated materials. Numerically, the costly parts are the evaluation of the fRG differential flow equations, usually determined by Feynman-type loop diagrams, and the high number of components of the flowing functions such as $V_{\Lambda}\left(k_{1}, k_{2}, k_{3}, k_{4}\right)$.

Initially, the fRG in the form used here was developed and employed in 2D Hubbard models in view of high- $T_{c}$ cuprates. Early works using so-called $N$-patch techniques to resolve the emerging wave-vector dependence of the flowing interaction focused on the exploration of the leading-ordering tendencies of the model [7-10] and understanding similarities to onedimensional models. This included the question of whether the pseudogap of the cuprates is foreshadowed in the fRG flow of weak to moderately coupled Hubbard models [11]. Parallel developments were conducted in the field of inhomogeneous one-dimensional systems [33,34]. Already at early stages, they were able to perform some quantitative benchmarking with exact numerical techniques (density matrix renormalization group) and to include partial self-energy corrections in the fRG. For 2D models, incorporating the fRG flow of the self-energy was technically more difficult for a number of reasons [11], but works indicating the opening of pseudogaps in the 2D flows came out a few years later $[35,36]$. However, at that time, few attempts were made to assess the quantitative precision of the method in comparison with other theoretical methods. Rather, the $N$-patch fRG techniques were used in numerous applications to broad classes of experimentally relevant material systems, such as the iron superconductors [37,38], graphene [39-41], and in the search for interactioninduced topological states $[42,43]$. There, the fRG was mainly used as a flexible tool to explore ground-state phase diagrams over a wide range of parameters.

Besides these applications, the formal development saw a steady evolution. Recently, the long-standing challenge of relating fRG schemes to the parquet approach was addressed $[44,45]$. Within the so-called multiloop extension of the fRG, all higher-loop contributions to the flow of parquet diagrams are accounted for with their exact weight. Conveniently, the effort only grows linearly with the loop order. The equivalence between the parquet approximation (PA) and multiloop fRG has been rigorously established at the level of spinless [44] and spinful [46] impurity models. Its numerical verification in the 2D Hubbard model poses additional challenges, due to the necessity of additionally treating the 2D momentum variables. Specifically, we will demonstrate how it is possible to render the numerical effort for a quantitative treatment of the fRG flow manageable in $2 \mathrm{D}$, by exploiting the multichannel decomposition [47] of the interaction vertex in combination with form-factor expansions [41,48]. This frees resources to incorporate frequency dependences and self-energy corrections in various cases $[49,50]$ and to conduct flows into symmetry-broken states [51,52]. Strongly inspired by earlier channel-decomposition schemes [48] and the singular-mode fRG by Wang et al. [41], the so-called truncated unity fRG was set up [53]. This formalism combines various technical improvements and allows for the development of a highly parallelizable and fast-performing code, mainly involving 2D (or 3D) integrations and matrix multiplications [54]. The name "truncated unity" comes from the insertion of unity into loop diagrams. These unities are sums over form factors, which are truncated by only considering the relevant form factors. As the form factors can be related to fermion bilinears on the real-space lattice, one obtains a physically appealing understanding of what the given truncation captures and what is left out. Furthermore, one can check the convergence of the truncation by varying the number of form factors kept [53]. Notably, the truncated unity fRG methodology is useful in another diagrammatic approach, the parquet scheme, whose performance can be boosted significantly in the truncated unity PA [55,56]. A major step forward was achieved by including the frequency dependence and self-energy corrections into the truncated unity fRG framework [57] and related schemes [58,59]. Supplemented by multiloop corrections [57], this paved the way for (a) internal convergence of the fRG as a function of the number of loops and (b) internal consistency in the fRG, as different ways to compute response functions (by the flow of external-field couplings or by postprocessing of the final interaction vertex) and flows with different cutoff schemes are found to agree.

In this paper we now aim at demonstrating the accuracy of our approach to describe one of the most challenging quantum many-body models of correlated electrons: the 2D Hubbard model. We do this by comparing data obtained by the truncated unity fRG in the multiloop extension (hereafter denoted by RRG $^{*}$ with the adapted self-energy flow and by fRG with the conventional self-energy flow) with other numerical methods. Going beyond the preliminary results of Ref. [57], restricted to the calculation of static response functions, we address here the computation of the self-energy in detail. In particular, investigating the form of the multiloop flow equation for the self-energy, we find that a form-factor expansion of the two-particle vertex prevents the full reconstruction of the Schwinger-Dyson equation (SDE) $[60,61]$ at loop convergence. The deviations can be traced back to the approximations introduced by the form-factor projections in the different channels and, more importantly, can be cured by using the direct derivative of the SDE instead. Including this methodological improvement, we provide a detailed analysis of the quantitative reliability of the fRG for the 2D Hubbard model by verifying the agreement with the solution of the PA. Further, in a comparison to quantum Monte Carlo data, we show that the fRG is remarkably accurate up to moderate interaction strengths. This demonstration may have considerable impact as it shows that the fRG can be pushed to become a quantitative quantum many-body method. One of its benefits for condensed matter material research is the unbiased treatment of various fluctuation channels down to low-energy scales, including the indication of ordering transitions and (pseudo)gap openings. This important feature is accompanied by the conceptual transparency of the approach that can be exploited to pin down the processes responsible for emerging physical effects. From a longer-term perspective, a quantitatively reliable implementation of the fRG approach provides the most suitable setup for its combination $[62,63]$ with complementary quantum many-body approaches, such as the dynamical mean-field theory (DMFT). The improvements described in this paper could thus represent an essential step for an accurate description of two-dimensional 
electron systems even in the intermediate- to strong-coupling regime.

The paper is organized as follows. In Sec. II we introduce the Hubbard model and the main observables. In Sec. III we discuss the multiloop flow equation of the self-energy and present its extension in the truncated unity fRG framework, showing that it correctly accounts for the form-factor projections in the different channels. Section IV contains a brief description of our benchmarking methods: the PA and determinant quantum Monte Carlo (DQMC). In Secs. V and VI we illustrate our results for the self-energy and the susceptibilities for the 2D Hubbard model at half filling and out of it, provide numerical evidence for the convergence to the PA, and perform benchmarks with the DQMC data. We conclude with a summary and an outlook in Sec. VII.

\section{MODEL AND OBSERVABLES}

\section{A. Two-dimensional Hubbard model}

We consider the single-band Hubbard model in two dimensions,

$$
\hat{\mathcal{H}}=\sum_{i, j, \sigma} t_{i j} \hat{c}_{i \sigma}^{\dagger} \hat{c}_{j \sigma}+U \sum_{i} \hat{n}_{i \uparrow} \hat{n}_{i \downarrow}-\mu \sum_{i, \sigma} \hat{n}_{i \sigma},
$$

where $\hat{c}_{i \sigma}\left(\hat{c}_{i \sigma}^{\dagger}\right)$ annihilates (creates) an electron with spin $\sigma$ at the lattice site $i\left(\hat{n}_{i \sigma}=\hat{c}_{i \sigma}^{\dagger} \hat{c}_{i \sigma}\right), t_{i j}=-t$ is the hopping between nearest-neighbor sites, $t_{i j}=-t^{\prime}$ is the hopping between next-nearest-neighbor sites, $\mu$ is the chemical potential, and $U$ is the on-site Coulomb interaction. The bare propagator is

$$
G_{0}(\mathbf{k}, i v)=\left(i v+\mu-\epsilon_{\mathbf{k}}\right)^{-1},
$$

with

$$
\epsilon_{\mathbf{k}}=-2 t\left(\cos k_{x}+\cos k_{y}\right)-4 t^{\prime} \cos k_{x} \cos k_{y} .
$$

In the following we use $t \equiv 1$ as the energy unit.

\section{B. Susceptibilities and self-energy}

We compute different susceptibilities describing the linear response of a system to a weak external perturbation, as obtained from the $\mathrm{fRG}^{*}$, the PA, and DQMC. In Matsubara frequency space, the susceptibilities are defined via a Fourier transform with respect to imaginary time $\tau$,

$$
\chi_{\eta}(\mathbf{q}, i \omega)=\int_{0}^{\beta} d \tau e^{i \omega \tau} \chi_{\eta}(\mathbf{q}, \tau)
$$

where $\eta=\mathrm{M} / \mathrm{D} / \mathrm{SC}$ indicates the magnetic, density, and superconducting ( $s$ - and $d$-wave) channels, respectively.

In the half-filled Hubbard model the dominant susceptibility is the antiferromagnetic (AF) one, defined by $\chi_{\mathrm{AF}}=$ $\chi_{\mathrm{M}}(\mathbf{q}=(\pi, \pi), i \omega=0)$ through the magnetic (or spin) susceptibility

$$
\chi_{\mathrm{M}}(\mathbf{q}, \tau)=\left\langle T_{\tau} \hat{s}^{z}(\mathbf{q}, \tau) \hat{s}^{z}(\mathbf{q}, 0)\right\rangle-\left\langle\hat{s}^{z}(\mathbf{q}, \tau)\right\rangle\left\langle\hat{s}^{z}(\mathbf{q}, 0)\right\rangle,
$$

where we use the spin operator in the $z$ direction $\hat{s}^{z}(\mathbf{q}, \tau)=$ $\left[\hat{n}_{\uparrow}(\mathbf{q}, \tau)-\hat{n}_{\downarrow}(\mathbf{q}, \tau)\right] / 2$ and the spin-resolved density operator $\hat{n}_{\sigma}(\mathbf{q}, \tau)=\sum_{\mathbf{k}} \hat{c}_{\sigma}^{\dagger}(\mathbf{k}+\mathbf{q}, \tau) \hat{c}_{\sigma}(\mathbf{k}, \tau)$. The sum over momenta includes the normalization factor associated with the momentum integration (or summation) over the first Brillouin zone.
The density (or charge) response function is defined by

$$
\chi_{\mathrm{D}}(\mathbf{q}, \tau)=\frac{1}{4}\left(\left\langle T_{\tau} \hat{n}(\mathbf{q}, \tau) \hat{n}(\mathbf{q}, 0)\right\rangle-\langle\hat{n}(\mathbf{q}, \tau)\rangle\langle\hat{n}(\mathbf{q}, 0)\rangle\right),
$$

with $\hat{n}(\mathbf{q}, \tau)=\hat{n}_{\uparrow}(\mathbf{q}, \tau)+\hat{n}_{\downarrow}(\mathbf{q}, \tau)$. In particular, we will show results for the charge compressibility $\kappa=4 \chi_{\mathrm{D}}(\mathbf{q}=$ $(0,0), i \omega=0)$ and the charge density wave susceptibility $\chi_{\mathrm{CDW}}=\chi_{\mathrm{D}}(\mathbf{q}=(\pi, \pi), i \omega=0)$.

For the $n=s, d$ pairing susceptibility

$$
\begin{aligned}
\chi_{\mathrm{SC}, n}(\mathbf{q}, \tau)= & \frac{1}{4}\left\langle T_{\tau}\left[\hat{\Delta}_{n}^{\dagger}(\mathbf{q}, \tau)+\hat{\Delta}_{n}(\mathbf{q}, \tau)\right]\right. \\
& \left.\times\left[\hat{\Delta}_{n}^{\dagger}(\mathbf{q}, 0)+\hat{\Delta}_{n}(\mathbf{q}, 0)\right]\right\rangle,
\end{aligned}
$$

we consider both the local $s$-wave pairing $\hat{\Delta}_{S}(\mathbf{q}, \tau)=$ $\sum_{\mathbf{k}} \hat{c}_{\uparrow}^{\dagger}(\mathbf{q}-\mathbf{k}, \tau) \hat{c}_{\downarrow}^{\dagger}(\mathbf{k}, \tau)$ and the nearest-neighbor $d$-wave pairing $\hat{\Delta}_{d}(\mathbf{q}, \tau)=\sum_{\mathbf{k}}\left[\cos \left(k_{x}\right)-\cos \left(k_{y}\right)\right] \hat{c}_{\uparrow}^{\dagger}(\mathbf{q}-$ $\mathbf{k}, \tau) \hat{c}_{\downarrow}^{\dagger}(\mathbf{k}, \tau)$. We will focus on the $\mathbf{q}=(0,0)$ and $i \omega=0$ components, referred to as $\chi_{\mathrm{SC}, s}$ and $\chi_{\mathrm{SC}, d}$, respectively.

Furthermore, we compute the self-energy $\Sigma(\mathbf{k}, i v)=$ $G_{0}^{-1}(\mathbf{k}, i v)-G^{-1}(\mathbf{k}, i v)$, where $G$ is the renormalized propagator $G(\mathbf{k}, \tau)=-\left\langle T_{\tau} \hat{c}_{\sigma}(\mathbf{k}, \tau) \hat{c}_{\sigma}^{\dagger}(\mathbf{k}, 0)\right\rangle$. We also show results for the double occupancy (DOC), which can be obtained either from the susceptibilities

$$
\mathrm{DOC}^{(2 \mathrm{P})}=\sum_{i \omega} \int d \mathbf{q}\left[\chi_{\mathrm{D}}(\mathbf{q}, i \omega)-\chi_{\mathrm{M}}(\mathbf{q}, i \omega)\right]+n_{\uparrow} n_{\downarrow}
$$

at the two-particle level or, equivalently, from

$$
\operatorname{DOC}^{(1 \mathrm{P})}=\frac{1}{U} \sum_{i \nu} e^{i v 0^{+}} \int d \mathbf{k} \Sigma(\mathbf{k}, i v) G(\mathbf{k}, i v)
$$

at the one-particle level. The sum over frequencies includes the normalization factor of temperature $T$.

\section{MULTILOOP fRG}

\section{A. Channel decomposition of the vertex}

Next to the self-energy, a central object of fRG and parquet algorithms is the (one-particle irreducible) two-particle vertex $F$. Using the SU(2) spin symmetry [21], we can restrict ourselves to one spin component $V=F^{\uparrow \uparrow \downarrow \downarrow}$. From the vertex, the susceptibilities can be computed by contracting the two-particle vertex at the end of the flow (postprocessed) or alternatively via the flow of the response vertices [57] (see Appendix A for a more detailed discussion).

In the channel or parquet decomposition of the vertex we can identify the two-particle reducible contributions $\Phi_{p p / p h / \overline{p h}}$ in the particle-particle, particle-hole, and crossed (or transverse) particle-hole channels, respectively. We have

$$
\begin{aligned}
V\left(k_{1}, k_{2}, k_{3}, k_{4}\right)= & \Lambda_{2 \mathrm{PI}}+\Phi_{p h}\left(k_{2}-k_{1}, k_{1}, k_{4}\right) \\
& +\Phi_{\overline{p h}}\left(k_{3}-k_{2}, k_{1}, k_{2}\right) \\
& +\Phi_{p p}\left(k_{1}+k_{3}, k_{1}, k_{4}\right),
\end{aligned}
$$

where the reducible vertices on the right-hand side (rhs) are parametrized according to a single generalized transfer momentum and two fermionic momenta. In the parquet approximation, the fully two-particle irreducible vertex is approximated by $\Lambda_{2 \mathrm{PI}}=U$. Fully accounting for the interplay between different two-particle channels is a central motivation for the multiloop extension of the fRG, described next. 


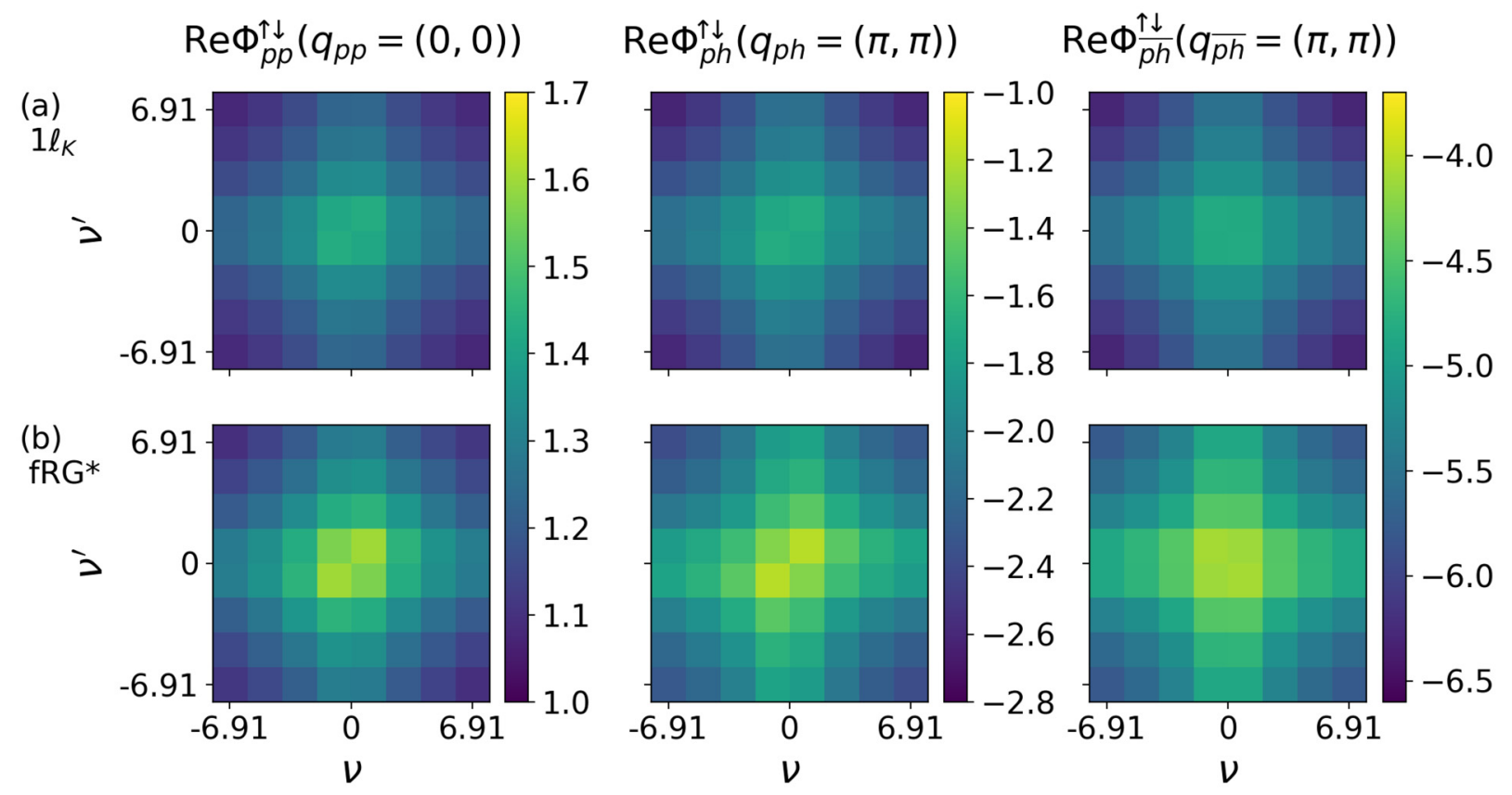

FIG. 1. Two-particle vertex decomposed as in Eq. (10) in the different $s$-wave channel contributions at zero bosonic frequency as a function of fermionic frequencies: (a) $1 \ell$ including the katanin correction vs (b) multiloop data, for $U=2$ and $1 / T=5$.

\section{B. Multiloop extension of the fRG: A brief overview}

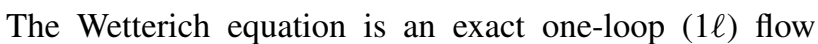
equation for the generating functional of one-particle irreducible vertices [15]. Expanding in the vertices leads to an infinite hierarchy of one-loop flow equations for the vertices. However, objects like the three-particle vertex are intractable for numerical treatments. The fundamental approximation in many fRG flows is therefore the truncation in the hierarchy of flow equations [64]. Setting the three-particle vertex to zero yields an approximate $1 \ell$ flow equation for the selfenergy and two-particle vertex. A common way to reintroduce some of the lost contributions is to reuse the scale derivative of the self-energy $\dot{\Sigma}$ in the flow of the two-particle vertex. This Katanin substitution [65] already leads to significantly improved results, labeled by $1 \ell_{K}$ in the following. A further refinement, which effectively incorporates the three-particle vertex to third order in the renormalized interaction, is realized by reusing the $1 \ell$ results in a $2 \ell$ addition to the vertex flow $[51,65,66]$.

The multiloop fRG [44,45] extends these schemes to arbitrary loop order. One finds that the multiloop additions complete the scale derivative of all the diagrams which are only partly generated in the $1 \ell$ flow. Thus, they remove the dependence of the results from the particular choice of the regulator; the quantitative reliability of these results is the subject of the present paper. Due to the iterative structure of the multiloop corrections, the numerical effort grows linearly with the loop order. Starting from an efficient algorithm for the $1 \ell$ flow, the implementation of the multiloop equations is straightforward.

An alternative derivation of the multiloop flow equations [67] highlights the close connection between the fRG and the parquet approach (see also Sec. III C). Starting from the parquet equations, a scale dependence of propagators and vertices can be introduced by making the bare propagator scale dependent. Taking the scale derivative of the selfconsistent parquet equations then leads to the multiloop flow equations. The equivalence is exact, provided the self-energy and vertices are treated without further approximation of their momentum and frequency dependence.

A crucial ingredient for such an equivalence and overall quantitative accuracy is a good resolution of the two-particle vertex and the self-energy in momentum and frequency space. Since the numerical effort grows very fast with the number of momentum patches and the size of the frequency window, an efficient vertex parametrization of the two-particle vertex is crucial. We use the truncated unity fRG $[53,68]$ for the momentum dependence together with an accurate treatment of the frequency dependence which includes the high-frequency asymptotics [69]. For a detailed description and their implementation in the multiloop extension we refer to Refs. [57,70], where we also provide the expression of the employed smooth frequency cutoff. Here we further use a refined momentum grid to resolve the peak at $\mathbf{q}=(\pi, \pi)$ in the AF susceptibility at half filling. This can be easily accounted for in the truncated unity formulation with precalculated projection matrices in real space [57]. The accurate description of the long-range AF fluctuations is also of major importance in order to fulfill the Mermin-Wagner theorem [71].

The effect of the multiloop extension on the frequency structure of the two-particle vertex $V$ is exemplified in Fig. 1 . Here we compare $1 \ell_{K}$ results with fully converged fRG* results, where the latter are obtained by using the revised multiloop flow equation of the self-energy presented in Sect. III D. While $\Phi_{p p}$ is enhanced in the fRG*, $\Phi_{p h}$ and $\Phi_{\overline{p h}}$ are screened and their absolute values are smaller. The screening of $\Phi_{\overline{p h}}$ with more loops is also reflected in the AF susceptibility, shown in Fig. 2, which is dominated by the contribution of the magnetic channel $\Phi_{M}=-\Phi_{\overline{p h}}$. The $1 \ell$ scheme strongly 


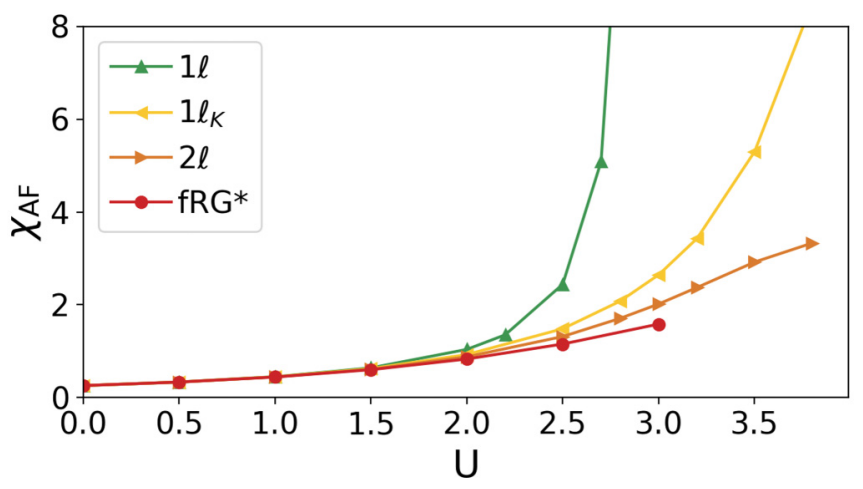

FIG. 2. Antiferromagnetic susceptibility $\chi_{\mathrm{AF}}(i \omega=0)$ defined in Eq. (5) as a function of the bare interaction $U$ for $1 / T=5$.

overestimates the peak at momentum transfer $\mathbf{q}=(\pi, \pi)$ leading to an $\mathrm{AF}$ ordering at finite interaction strength in violation of the Mermin-Wagner theorem [71]. With increasing loop order, the AF peak is reduced. At an inverse temperature of $1 / T=5$, the $2 \ell$ result is already very close to the fRG* on the scale of this plot. We note that for $U=3$ the fRG* is not fully converged with respect to frequencies and loop order, and for this reason no results for larger values of $U$ are displayed. The convergence threshold we use is $1 \%$ for $\chi_{\mathrm{AF}}$ and for $\operatorname{Im} \Sigma$ at momenta $(\pi, 0)$ and $(\pi / 2, \pi / 2)$ and the first two Matsubara frequencies. For completeness, we report the parameters used for the benchmark analysis of Secs. V and VI in Appendix B.

\section{Parquet approximation and postprocessing of the self-energy}

Generally, in many-body theory, there are various exact relations between one- and two-particle quantities, such as the Bethe-Salpeter equations [72,73] connecting different parts of the two-particle vertex and the SDE relating the selfenergy and vertex. While these equations are used in parquet approaches to iteratively find a self-consistent solution on the one- and two-particle level, applying them to the final self-energy and vertex of any method is a way to check its consistency.

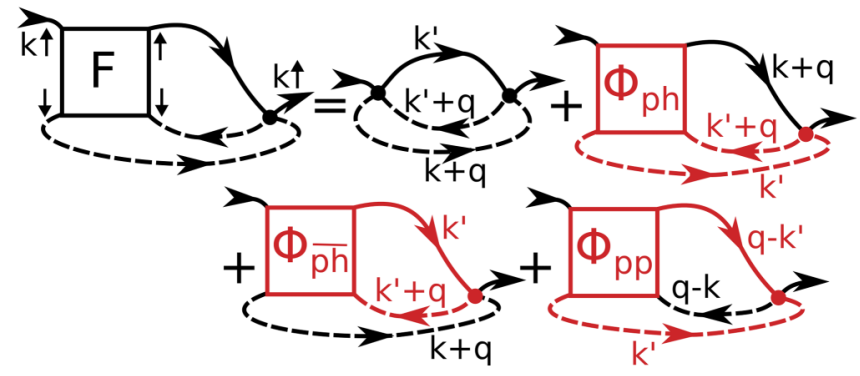

FIG. 3. Right-hand side of the Schwinger-Dyson equation for the self-energy (12), illustrating the contributions of the different channels, where solid (dashed) lines carry spin up (down). The first diagram on the rhs can be calculated using the convolution theorem and fast-Fourier-transform algorithms. The other contributions can be determined by first combining the two-particle reducible vertices $(\Phi)$ and the bare vertex (dot) through the propagator pair of the corresponding channel (red). Finally, each diagram is closed by a propagator (black) through the direct summation over frequency and momentum.

In our previous work [57], we focused on two-particle quantities like the susceptibilities and compared their outcome directly from the fRG flow with their postprocessed result using the final self-energy and vertex. Here we use the SDE to analogously determine the self-energy. The SDE involves the self-energy itself through the full propagator as well as the vertex and reads

$$
\begin{aligned}
\Sigma(\mathbf{k}, i v)= & -\sum_{\mathbf{k}^{\prime} \mathbf{q}} \sum_{i v^{\prime} i \omega} V\left(\mathbf{k}, \mathbf{k}^{\prime}, \mathbf{k}^{\prime}+\mathbf{q}, i v, i v^{\prime}, i v^{\prime}+i \omega\right) \\
& \times G\left(\mathbf{k}^{\prime}, i v^{\prime}\right) G\left(\mathbf{k}^{\prime}+\mathbf{q}, i v^{\prime}+i \omega\right) \\
& \times G(\mathbf{k}+\mathbf{q}, i v+i \omega) U .
\end{aligned}
$$

Note that the fourth dependence of the vertex is determined by momentum and frequency conservation. Its diagrammatic representation is shown on the left-hand side of Fig. 3. Note that we take the Hartree part implicitly into account by shifting the chemical potential by $U\left\langle\hat{n}_{\sigma}\right\rangle$; half filling then corresponds to $\mu=0$.

In the parquet decomposition (10), the vertex is split into the fully irreducible part and the three two-particle channels. As depicted on the right-hand side of Fig. 3, the SDE is then determined by four parts

$$
\begin{aligned}
\Sigma(\mathbf{k}, i v)= & -\sum_{\mathbf{k}^{\prime} \mathbf{q}} \sum_{i v^{\prime} i \omega} U^{2} G\left(\mathbf{k}^{\prime}, i v^{\prime}\right) G\left(\mathbf{k}^{\prime}+\mathbf{q}, i v^{\prime}+i \omega\right) G(\mathbf{k}+\mathbf{q}, i v+i \omega) \\
& +\sum_{\mathbf{q} i \omega} \sum_{m} f_{m}^{*}(\mathbf{k})\left[\sum_{i v^{\prime}} \sum_{n} \Phi_{p h, m n}\left(\mathbf{q}, i \omega, i v, i v^{\prime}\right) \Pi_{p h, n 0}\left(\mathbf{q}, i \omega, i v^{\prime}\right) 4 \pi^{2} U f_{0}(\mathbf{k})\right] G(\mathbf{k}+\mathbf{q}, i v+i \omega) \\
& +\sum_{\mathbf{q} i \omega} \sum_{m} f_{m}^{*}(\mathbf{k})\left[\sum_{i v^{\prime}} \sum_{n} \Phi_{\overline{p h, m n}}\left(\mathbf{q}, i \omega, i v, i v^{\prime}\right) \Pi_{p h, n 0}\left(\mathbf{q}, i \omega, i v^{\prime}\right) 4 \pi^{2} U f_{0}(\mathbf{k})\right] G(\mathbf{k}+\mathbf{q}, i v+i \omega) \\
& -\sum_{\mathbf{q} i \omega} \sum_{m} f_{m}^{*}(\mathbf{k})\left[\sum_{i v^{\prime}} \sum_{n} \Phi_{p p, m n}\left(\mathbf{q}, i \omega, i v, i v^{\prime}\right) \Pi_{p p, n 0}\left(\mathbf{q}, i \omega, i v^{\prime}\right) 4 \pi^{2} U f_{0}(\mathbf{k})\right] G(\mathbf{q}-\mathbf{k}, i \omega-i v),
\end{aligned}
$$


where $f_{n}(\mathbf{k})$ are the form factors in the truncated unity fRG. The first diagram can be calculated in real space using the convolution theorem twice. The remaining parts in the $p h-, \overline{p h}$-, and $p p$ channels involve

$$
\begin{aligned}
& \Pi_{p h, n m}(\mathbf{q}, i \omega, i v)=-\int d \mathbf{p} f_{n}^{*}(\mathbf{p}) f_{m}(\mathbf{p}) G(\mathbf{p}, i v) G(\mathbf{q}+\mathbf{p}, i \omega+i v), \\
& \Pi_{p p, n m}(\mathbf{q}, i \omega, i v)=\int d \mathbf{p} f_{n}^{*}(\mathbf{p}) f_{m}(\mathbf{p}) G(\mathbf{p}, i v) G(\mathbf{q}-\mathbf{p}, i \omega-i v),
\end{aligned}
$$

where we used the same conventions as in Ref. [57].

Upon applying Eq. (12) as a postprocessing procedure to compute the self-energy at the end of the fRG flow, we find deviations of up to $20 \%$ in both the real and the imaginary part with respect to the solution of the conventional flow equation

$$
\begin{aligned}
\dot{\Sigma}^{\Lambda}(k)= & \sum_{k^{\prime}}\left[2 V^{\Lambda}\left(k, k, k^{\prime}\right)-V^{\Lambda}\left(k^{\prime}, k, k\right)\right] S^{\Lambda}\left(k^{\prime}\right) \\
& +\dot{\Sigma}_{\text {mfRG-corr }}
\end{aligned}
$$

Here the first lines corresponds to the $1 \ell$ flow equation and the second to the multiloop corrections [45]. While at $1 \ell$, a difference between the flowing and the postprocessed result is not surprising, we expect these differences to vanish in a (loop) converged multiloop fRG solution. As we will show in the following, the remaining discrepancies originate from the truncated form-factor expansion, for which the flow of the self-energy has to be replaced by the direct derivative of the SDE. ${ }^{1}$

\section{Self-energy flow in a form-factor expansion}

The different $\mathrm{fRG}$ results for the self-energy obtained from the (multiloop) flow and the postprocessing via the SDE can be traced back to the truncated unity treatment with a reduced number of form factors. ${ }^{2}$ In this case, some of the identities used in the general derivation of the self-energy flow [67] do not hold anymore. Consequently, the approximation of the vertex in terms of form factors destroys the equivalence of the flow equation and the SDE. However, this problem can be overcome by using the direct derivative of the SDE instead.

In more detail, each summand of the SDE contains two vertices and three propagators. In Ref. [67], multiple transformations which interchange these propagators have been used, amounting to a translation between the different two-particle channel descriptions. However, in the truncated unity parametrization, channel transformations are only information-loss-free in the infinite form-factor limit. With a finite number of form factors, the invariance of the SDE under the exchange of propagators does not hold anymore.

Here we propose the fRG* extension which exactly reproduces the SDE for the self-energy in a form-factor expansion of the two-particle vertex. Technically, the flow of the self-energy is replaced by the direct derivative of the SDE (for details see Appendix $C$ ), which can be found by first introducing a scale $(\Lambda)$ dependence in the SDE and then taking the derivative with respect to $\Lambda$ [67].

\footnotetext{
${ }^{1}$ For a related fRG scheme starting from the SDE, see Ref. [74].

${ }^{2}$ In the limit of an infinite number of form factors the differences would vanish.
}

An example illustrating the advantage of the new fRG* self-energy flow compared to the conventional flow is shown in Fig. 4. We focus on two specific differentiated diagrams contributing to the flow of the self-energy. In the fRG*, each summand of the SDE is directly differentiated with respect to $\Lambda$. For concreteness, we consider the penultimate summand on the rhs of Fig. 3, insert the lowest-order diagram for $\Phi_{\overline{p h}}$, and let the $\Lambda$ derivative act on the two spin-down propagators (dashed lines). Thereby, we obtain the two differentiated selfenergy diagrams on the rhs of Fig. 4(a), where the propagators with a diagonal dash symbolize differentiated propagators. The same contributions should be part of the standard fRG self-energy flow, shown in Fig. 4(b). Indeed, the first diagram on the rhs simply follows from a second-order $\Phi_{\overline{p h}}$ diagram with two $\overline{p h}$ bubbles and the second one originates from the $\Phi_{p p}$ part of $F$, where the $\overline{p h}$ bubble (orange) is inserted into another $p p$ bubble (blue). The crucial point is that the right-hand sides of both panels are formally equivalent, but the form-factor truncation applies in a less favorable way on the right diagram in the fRG [Fig. 4(b)]: After each colored bubble, a truncated unity projects the dependence on the fermionic momenta onto a finite number of form factors. The two diagrams in Fig. 4(a) and the first in Fig. 4(b) are exactly described by only $s$-wave form factors. However, when evaluating the diagram in Fig. 4(b) in an $s$-wave formfactor truncation, the $\overline{p h}$ contribution is completely averaged in the process of translating it to the $p p$ channel, thus yielding significantly less accurate results.

In Fig. 5 we present the real and imaginary parts of the self-energy $\Sigma(i v=i \pi T)$ as a function of momentum, for

(a)

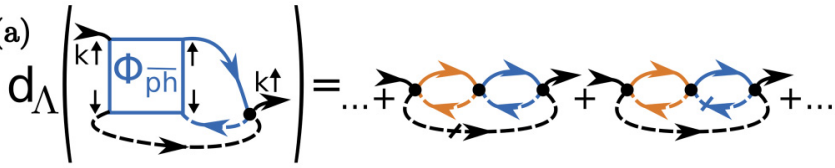

(b)

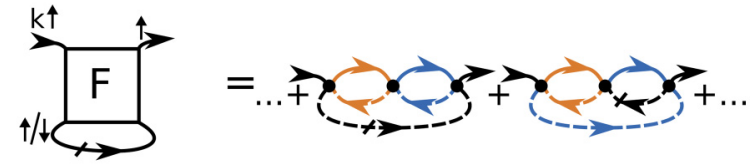

FIG. 4. Illustration of the self-energy flows in (a) the fRG*, restricted to the part with $\Phi_{\overline{p h}}$, and (b) the fRG. The rhs shows two exemplary differentiated diagrams contributing to the self-energy flow at third order, where solid (dashed) lines carry spin up (down) and the diagonal dash symbolizes a scale-differentiated bare propagator. In the $\mathrm{fRG}^{*}$, two bubbles from the same channel (colored) are combined and then closed with the black line. By contrast, in the $\mathrm{fRG}$, the second diagram requires one to insert a $\overline{p h}$ (orange) into a $p p$ (blue) bubble, before closing with the differentiated propagator (black). 

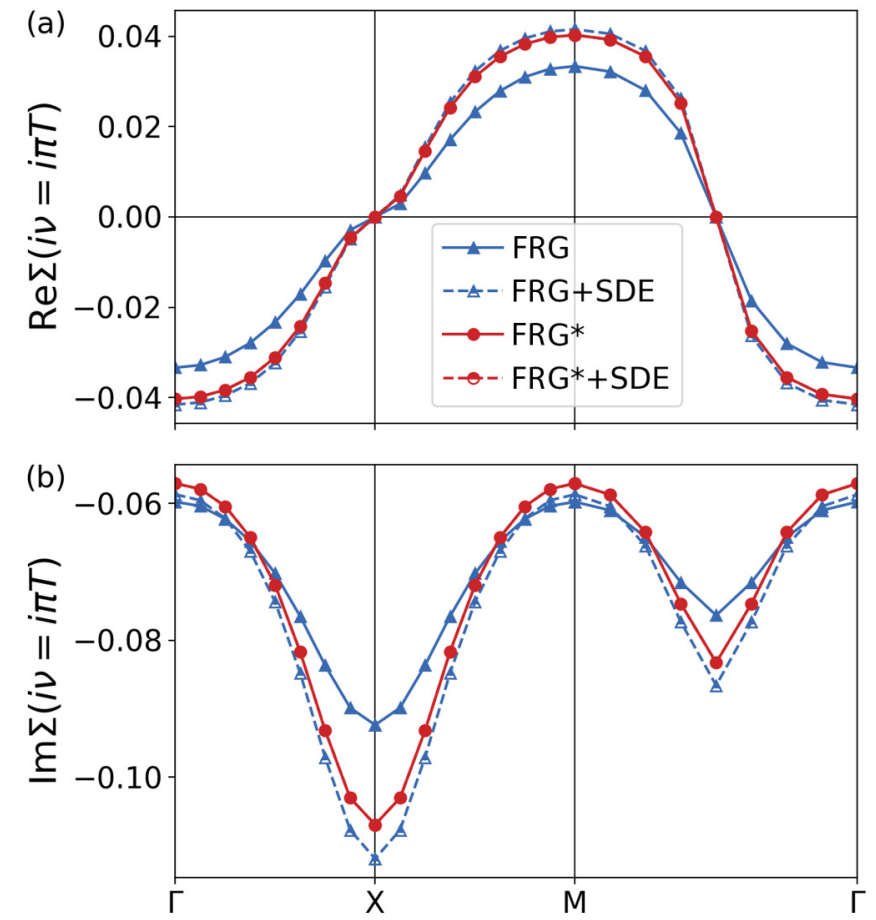

FIG. 5. (a) Real and (b) imaginary parts of the self-energy as obtained by the conventional fRG (blue) and the fRG* flow (red), together with the respective postprocessed results (dashed lines), for $U=2$ and $1 / T=5$. Within the $\mathrm{fRG}^{*}$, the postprocessed (dashed red) ones lie exactly on top of the fRG* flow results (red solid).

$U=2$ and $1 / T=5$, for both the conventional fRG (blue) and $\mathrm{fRG}^{*}$ (red). We show that, unlike the fRG, the fRG* yields excellent agreement between the flowing (solid lines) and the postprocessed self-energy (dashed lines), determined by Eq. (12) with the final vertices and self-energy at the end of the flow. The nonimproved fRG results obtained from the flow exhibit pronounced deviations with respect to the fRG* self-energy. For the postprocessed ones, these deviations are significantly reduced since in this case the self-energy is updated by the SDE at the end of the flow.

\section{E. Self-energy iterations}

We now analyze the effect of the self-energy iterations, i.e., a repeated [up to 5 times (see Appendix B)] evaluation of the rhs of the flow equation, in the fRG as well as in the $\mathrm{fRG}^{*}$. As described in detail in Appendix C, these are needed because the rhs of both the vertex and the self-energy depend on each other: In the vertex flow, the Katanin substitution $S(\mathbf{k}, i v)=\left.d_{\Lambda} G(\mathbf{k}, i v)\right|_{\dot{\Sigma}=0} \rightarrow S^{K}(\mathbf{k}, i v)=d_{\Lambda} G(\mathbf{k}, i v)$ depends on the self-energy flow, while the multiloop flow of $\Sigma$ involves the vertex flow, either through the multiloop corrections of Ref. [45] or through the parts of the differentiated SDE where $d_{\Lambda}$ acts on a vertex. In order to study the effect of the self-energy iterations, we compare the fully converged results to those obtained by solely using the $1 \ell$ self-energy flow in the Katanin substitution. Figure 6 displays self-energy results with (solid lines) and without (dotted lines) iterations, as obtained by the fRG (blue) and fRG* (red). We note that the slight kink between the fourth and fifth frequencies and
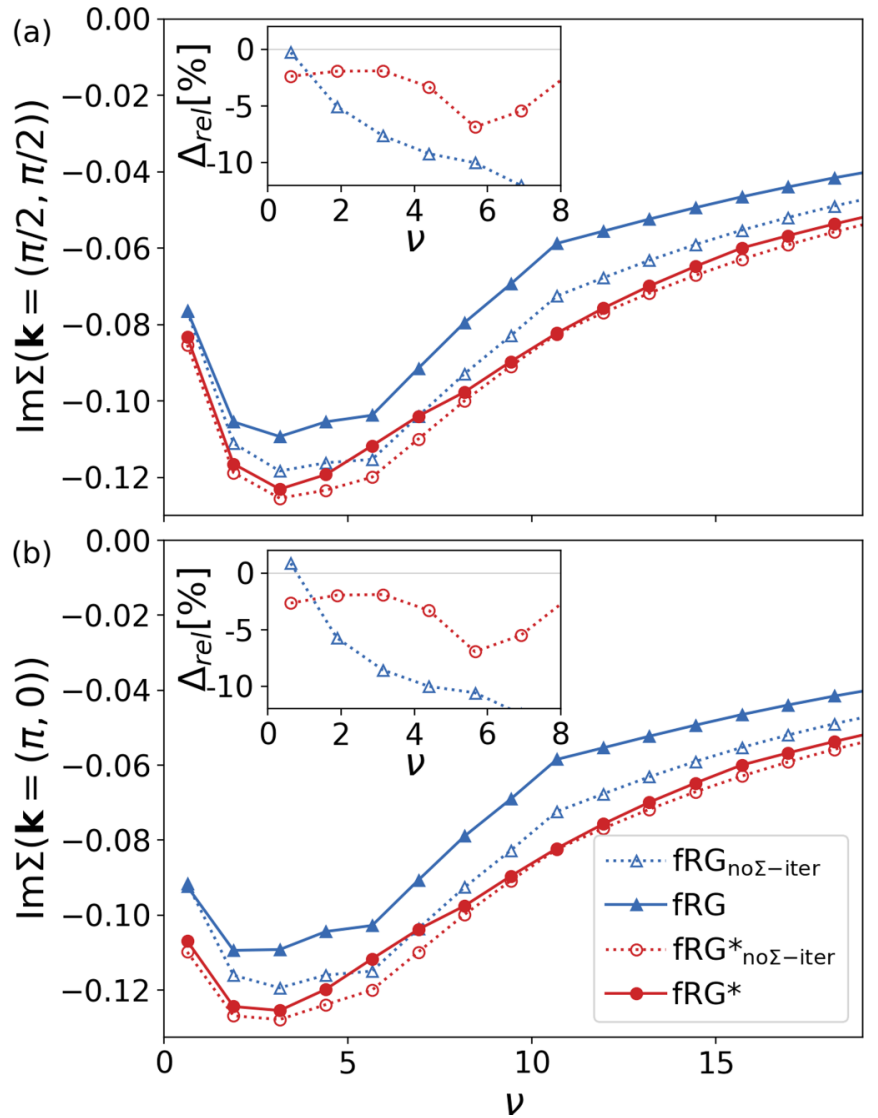

FIG. 6. Imaginary part of the self-energy at (a) the nodal and (b) the antinodal point, as obtained by the fRG (blue) and fRG* (red), with and without self-energy iterations, for $U=2$ and $1 / T=5$. The inset shows the relative difference caused by neglected self-energy iterations.

also between the eighth and ninth frequencies corresponds to the crossing of the low-frequency tensor range and the highfrequency asymptotics of the two-particle vertex. This effect is more pronounced in the fRG than in the fRG*, since the channel-reducible vertices that directly enter the conventional flow (15) have a richer frequency dependence than those that are first combined with the bare interaction, as needed for the calculation of the SDE-inspired fRG* flow (12). In the conventional fRG flow, the effect of the self-energy iterations amounts to 5\%-10\% except for the first Matsubara frequency, which appears to be well described without any additional iterations. In contrast, in the $\mathrm{fRG}^{*}$, which accounts for the form-factor projections in the different channels, the selfenergy iterations lead to much smaller overall corrections but are relevant for the lowest frequencies (see the inset in Fig. 6).

The self-energy iterations also affect the AF susceptibility $\chi_{\mathrm{AF}}$ displayed in Fig. 7. For $U=2$ and $1 / T=5$, a sizable effect is only observed for zero Matsubara frequency, highlighted in the inset. Neglecting the self-energy iterations in the $\mathrm{fRG}^{*}$ (red dotted lines) overestimates the AF peak by $1 \%$. In the fRG, their impact on $\chi_{\mathrm{AF}}$ is smaller, according to the small effect on the first Matsubara frequency of the self-energy observed in Fig. 6. Anticipating the comparison of the fRG* (red) to the PA (gray) (see Fig. 8), we remark that, 


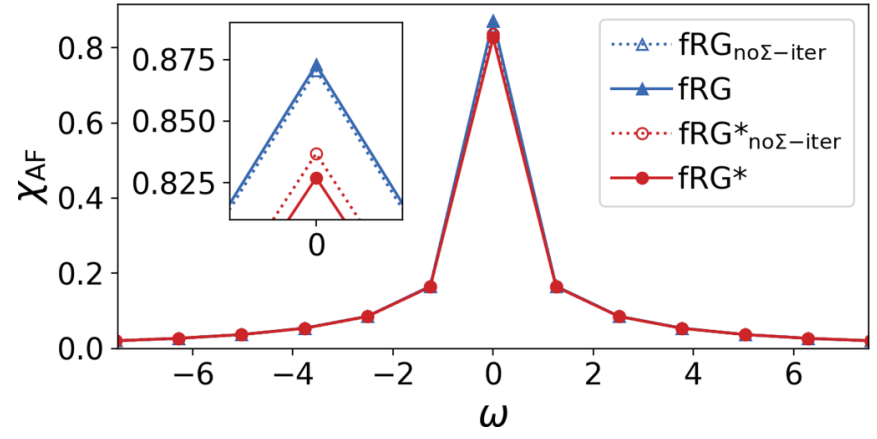

FIG. 7. Frequency dependence of the AF susceptibility $\chi_{\mathrm{AF}}(i \omega)$ as obtained by the conventional fRG (blue) and the fRG* flow (red), with (solid line) and without (dotted line) $\Sigma$ iteration, for $U=2$ and $1 / T=5$.

while the AF peak in the conventional scheme (blue) deviates by $5 \%$ from the PA, the $\mathrm{RRG}^{*}$ (red) shows perfect agreement.

\section{BENCHMARK METHODS}

\section{A. Parquet approximation}

The PA results are obtained with the truncated unity implementation of the parquet equations [56]. The parquet equations are solved by iterating the Bethe-Salpeter equations, the parquet equation (10), and the SDE (12) until self-consistency is reached. The momentum dependence of the vertices is parametrized using the form-factor expansion [55], identical to the scheme used in the truncated unity fRG. Although the equivalence of the PA and the multiloop fRG has been formally shown [67], the actual PA calculations substantially differ from the ones in the fRG, since no flow parameter is introduced. In the PA no differential equations are solved but the convergence to a fixed point is achieved iteratively, starting from an initial guess for the vertices (in our case given by the lowest-order diagrams).

In order to account for the finite-frequency box, we use the asymptotics as introduced in Ref. [75] and also used in Ref. [76]. The implementation of the frequency asymptotics thus differs from the one used in the fRG calculations. All further computational details of the truncated unity implementation of the parquet equations can be found in Ref. [56], as well as a detailed analysis of the convergence in the number of form factors, showing that the approximation of a single form factor, used here at half filling, is justified.

All results are converged in the number of discrete lattice momenta $N_{q}$ and positive fermionic Matsubara frequencies $N_{f+}$. Specifically, we use a uniform momentum grid with $N_{q}=32 \times 32$ and a frequency box with $N_{f+}=32$ positive Matsubara frequencies.

\section{B. Determinant quantum Monte Carlo}

The DQMC algorithm, proposed by Blankenbecler et al. [77], is a state-of-the-art numerically exact method and is commonly applied for finite-temperature $[77,78]$ calculations of interacting fermion systems. The basic idea of the DQMC algorithm is to decouple the two-body interaction into noninteracting fermions coupled with auxiliary fields and to

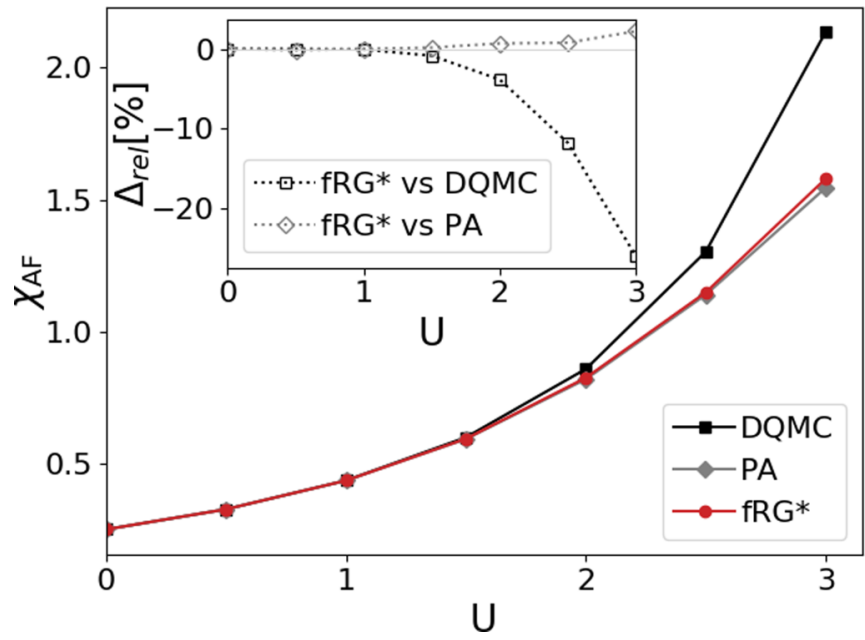

FIG. 8. Antiferromagnetic susceptibility $\chi_{\mathrm{AF}}$ as a function of $U$, as obtained by the fRG* (red), the PA (gray), and the DQMC (black), for $1 / T=5$. The inset shows the relative difference.

compute the fermionic observables via importance sampling of the fields. To achieve that, a Trotter decomposition and a Hubbard-Stratonovich transformation are successively used, after discretizing the inverse temperature as $\beta=M \Delta \tau$. The systematic error from finite $\Delta \tau$ can be removed by extrapolations with several different $\Delta \tau$ values. For further details, we refer to the reviews in $[79,80]$. In this work we choose $\Delta \tau t=0.02$, which has been tested to safely reach the $\Delta \tau \rightarrow$ 0 limit. In this work we have also implemented our most recent improvements $[81,82]$ of the DQMC algorithm. For the computation of dynamical quantities, we first measure the imaginary-time correlation functions and then obtain the imaginary-frequency observables via Fourier transformation. Specifically, for the self-energy we implemented the Legendre polynomial representation [83] for the imaginary-time singleparticle Green's function $G(\mathbf{k}, \tau)$ to compute $G(\mathbf{k}, i v)$ and subsequently applied the Dyson equation. This yields smooth self-energy results even for high frequencies. All DQMC data presented here are found to converge to the thermodynamic limit for a linear system size of $L=28$ (with the number of lattice sites being $N=L^{2}$ ) for half filling and $L=24$ away from it. As for statistics, we typically use in total $10^{5}$ measurement samples after the Markov chain equilibrium process. The error bars are significantly smaller than the corresponding symbol and thus neglected in the plots.

\section{RESULTS AT HALF FILLING}

We now compare different physically relevant quantities as obtained from the fRG*, the PA, and the numerically exact DQMC. In particular, we first focus on the various susceptibilities in Sec. VA and then present the results for the self-energy and double occupancy in Secs. VB and V C, respectively.

\section{A. Susceptibilities}

We first present the results for the leading AF susceptibility $\chi_{\mathrm{AF}}$ in the half-filled 2D Hubbard model as a function of the bare interaction strength $U$. In Fig. 8 we report the fRG* (red), 


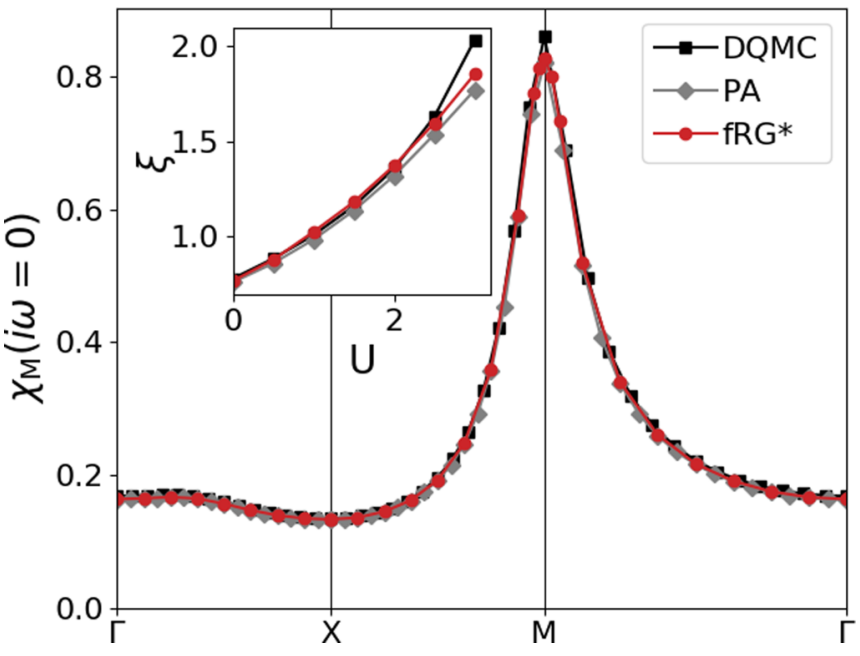

FIG. 9. Magnetic susceptibility $\chi_{M}(\mathbf{q}, i \omega=0)$ as obtained by the fRG* (red), the PA (gray), and the DQMC (black) for $U=2$ and $1 / T=5$. The inset shows the correlation length $\xi$ extracted from $\chi_{\mathrm{M}}(\mathbf{q}, i \omega=0)$ as a function of $U$ for $1 / T=5$ (see the text for details of the fitting procedure).

PA (gray), and DQMC data (black), together with the relative difference of the $\mathrm{fRG}^{*}$ with respect to the PA and DQMC shown in the inset. Up to $U=2.5$, the $\mathrm{fRG}^{*}$ and PA coincide with a relative difference less than or equal to $1 \%$. For larger values of $U$, the convergence of the $\mathrm{RRG}^{*}$ in frequencies and also in loop numbers becomes numerically challenging and is not reached yet (see also Appendix A). This leads to the observed deviations from the PA solution. The differences between the PA and the numerically exact DQMC data are essentially due to the fully two-particle irreducible diagrams not included in the PA. These diagrams contribute to fourth order in $U$; the corresponding relative difference amounts to $\Delta_{\text {rel }} \simeq 0.05 U^{4}$. A second source of the differences between the PA solution and the DQMC is given by the form-factor expansion of the two-particle vertex which accounts only for the local $s$-wave part. Due to perfect nesting, the physics at half filling is dominated by magnetic fluctuations peaked at $\mathbf{q}=(\pi, \pi)$, and at small coupling there are only minor quantitative corrections due to the form-factor truncation [56]. Away from half filling, we expect superconducting $d$-wave components to become relevant and hence include those form factors in Sec. VI too.

Figure 9 shows the momentum dependence of the magnetic susceptibility at zero frequency $\chi_{\mathrm{M}}(\mathbf{q}, i \omega=0)$ for $U=2$ (and $1 / T=5)$. The results of the $\mathrm{fRG}^{*}$, the PA, and the DQMC exhibit excellent quantitative agreement. The largest deviation is found at $M=(\pi, \pi)$ corresponding to the AF susceptibility shown in Fig. 8 for different values of $U$. We note that for all other frequencies $i \omega \neq 0$ the AF susceptibility of the $\mathrm{fRG}^{*}$ perfectly agrees with the one of the PA. While the AF peak height obtained from the $\mathrm{fRG}^{*}$ does not converge perfectly to the PA for $U>2$, the correlation length $\xi$ extracted from its width shows very good agreement between the different methods (see the inset in Fig. 9). The correlation length is fitted to all points of $\chi_{\mathrm{M}}(\mathbf{q}, i \omega=0)$ within a distance of $0.3 \pi$

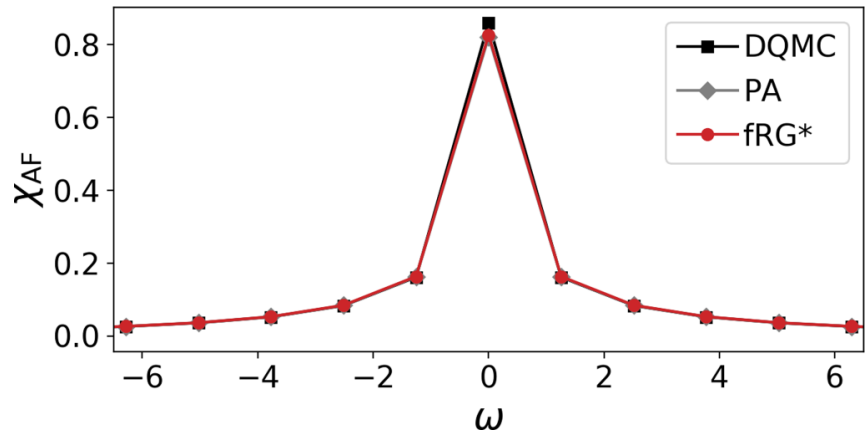

FIG. 10. Frequency dependence of the AF susceptibility $\chi_{\mathrm{AF}}(i \omega)$ as obtained by the fRG* (red), the PA (gray), and the DQMC (black) for $U=2$ and $1 / T=5$.

from $M$ through

$$
\chi(\mathbf{q}, i \omega=0) \sim \frac{1}{4 \sin ^{2}\left(\frac{q_{x}-\pi}{2}\right)+4 \sin ^{2}\left(\frac{q_{y}-\pi}{2}\right)+\xi^{-2}},
$$

which reduces to the Ornstein-Zernike form for small momentum differences $q_{x}-\pi$ and $q_{y}-\pi$ (cf. Refs. [84,85]). The number of momenta taken into account for the fit are between 33 and 45 in the fRG* ${ }^{*}$, between 69 and 161 in the PA, and 57 in the DQMC. The maximal standard deviation error is 0.023 in the $\mathrm{FRG}^{*}, 0.025$ in the PA, and 0.028 in the DQMC.

The frequency dependence of $\chi_{\mathrm{AF}}$ is shown in Fig. 10 for $U=2$ and $1 / T=5$. The $\mathrm{RRG}^{*}$ and PA compare very well at any Matsubara frequency; the largest deviation between the DQMC and $\mathrm{fRG}^{*}$ is found at zero Matsubara frequency.

In Fig. 11 we show different subleading susceptibilities as a function of $U$ for $1 / T=5$ : the compressibility $\kappa$, the $(s$ wave) charge density wave $\chi_{\mathrm{CDW}}$ susceptibility which equals $\chi_{\mathrm{SC}, s}$ for $\mathrm{SU}(2)$ spin and charge (particle-hole) symmetry (see Appendix E for the proof), and the $d$-wave superconducting susceptibility $\chi_{\mathrm{SC}, d}$. The quantitative agreement between the $\mathrm{fRG}^{*}, \mathrm{PA}$, and DQMC results for the subleading susceptibilities is very good, with the relative difference of the $\mathrm{fRG}^{*}$ with respect to the PA at $U=3$ being $10 \%$ for $\kappa, 4 \%$ for $\chi_{\mathrm{CDW}}$, and less than $1 \%$ for $\chi_{\mathrm{SC}, d}$ and with respect to the DQMC $15 \%$ for $\kappa, 6 \%$ for $\chi_{\mathrm{CDW}}$, and $2 \%$ for $\chi_{\mathrm{SC}, d}$. Note that the compressibility $\kappa$ is also consistent with Ref. [86]. The good agreement between the $\mathrm{RRG}^{*}$ and PA, both affected by the form-factor truncation, and the exact DQMC justifies a computation with only the local $s$-wave form factor.

All subleading susceptibilities $\kappa, \chi_{\mathrm{CDW}}, \chi_{\mathrm{SC}, s}$ and $\chi_{\mathrm{SC}, d}$ decrease with $U$, since the growing AF fluctuations lead to stronger screening of the subleading fluctuations. Figure 12 shows a more detailed analysis of the different contributions to the susceptibility and particularly of the importance of the vertex corrections. The uncorrelated susceptibilities in terms of dressed Green's functions (without vertex corrections) are determined by

$$
\begin{aligned}
\chi_{\mathrm{AF}, G G} & =\chi_{\mathrm{CDW}, G G} \\
& =\frac{1}{2} \sum_{i \nu} \Pi_{p h, 00}(\mathbf{q}=(\pi, \pi), i \omega=0, i \nu),
\end{aligned}
$$




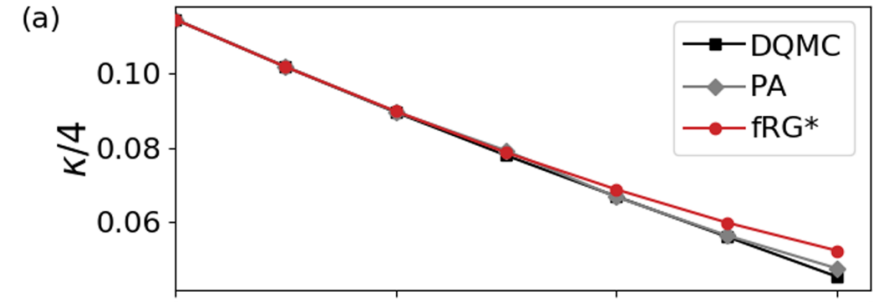

(b)

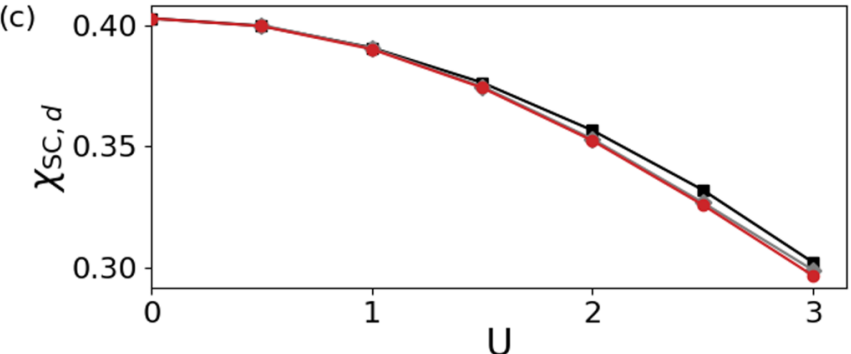

FIG. 11. (a) Compressibility $\kappa$, (b) charge density wave $\chi_{\mathrm{CDW}}=$ $\chi_{\mathrm{SC}, s}$, and (c) superconducting susceptibility $\chi_{\mathrm{SC}, d}(i \omega=0)$ as a function of $U$, as obtained by the fRG* (red), the PA (gray), and the DQMC (black), for $1 / T=5$.

$$
\begin{aligned}
\kappa_{G G} & =2 \sum_{i \nu} \Pi_{p h, 00}(\mathbf{q}=(0,0), i \omega=0, i \nu), \\
\chi_{\mathrm{SC}, d, G G} & =\frac{1}{2} \sum_{i \nu} \Pi_{p p, 11}(\mathbf{q}=(0,0), i \omega=0, i \nu),
\end{aligned}
$$

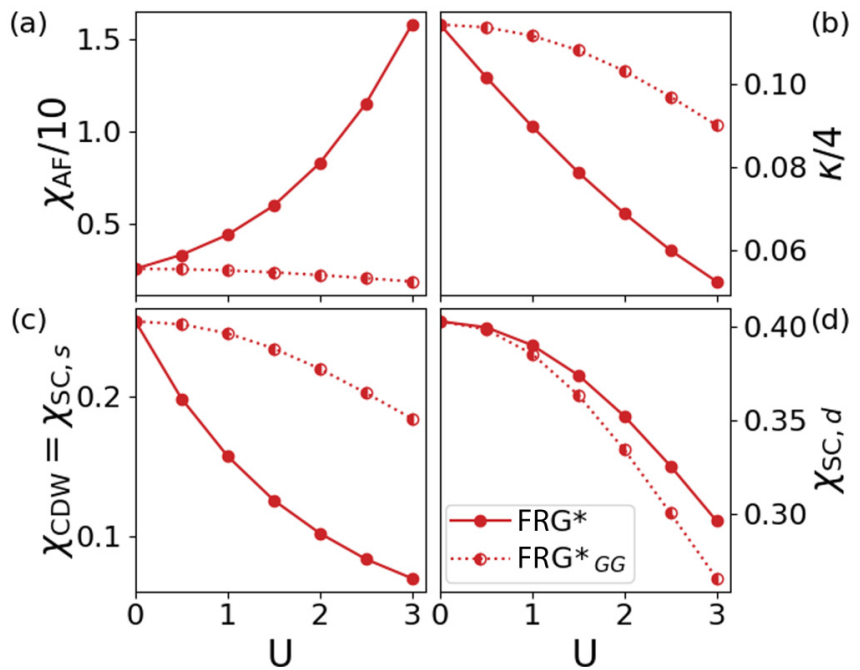

FIG. 12. (a) Antiferromagnetic susceptibility $\chi_{\mathrm{AF}}$, (b) compressibility $\kappa$, (c) charge density wave $\chi_{\mathrm{CDW}}=\chi_{\mathrm{SC}, s}$ (see Appendix B), and (d) $d$-wave superconducting susceptibility $\chi_{\mathrm{SC}}$ as a function of $U$, as obtained by the fRG* with (solid lines) and without (dashed lines) vertex corrections, for $1 / T=5$.

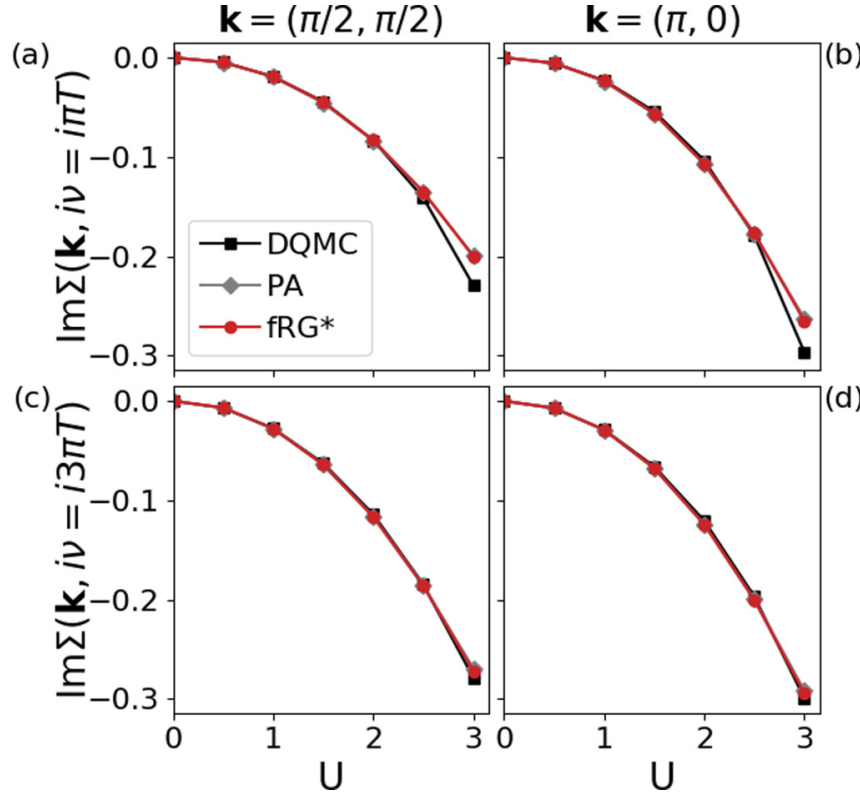

FIG. 13. Imaginary part of the self-energy at the (a) first frequency and nodal point, (b) first frequency and antinodal point, (c) second frequency and nodal point, and (d) second frequency and antinodal point as a function of $U$, as obtained by the $\mathrm{fRG}^{*}$ (red), the PA (gray), and the DQMC (black), for $1 / T=5$.

where the form-factor index 0 stands for the $s$ wave and 1 for the $d$ wave. They all decrease with $U$, as a consequence of self-energy screening effects. The vertex contributions, given by the difference from the full susceptibilities, exhibit a richer physical behavior: They lead to a reduction or screening of the bare $\kappa$ and $\chi_{\mathrm{SC}, s}$ susceptibilities, whereas $\chi_{\mathrm{SC}, d}$ and most prominently $\chi_{\mathrm{AF}}$ are enhanced with respect to their bare values. For $\chi_{\mathrm{SC}, d}$ the vertex corrections are not strong enough to induce an overall increasing susceptibility. This occurs only for $\chi_{\mathrm{AF}}$, where the vertex corrections are indeed dominant.

\section{B. Self-energy}

We now discuss the frequency and momentum dependence of the self-energy and their comparison between the different methods. In Fig. 13 we show the imaginary part at the nodal $\mathbf{k}=(\pi / 2, \pi / 2)$ and antinodal $\mathbf{k}=(\pi, 0)$ point as a function of $U$ for $1 / T=5$. The agreement between the fRG* and the PA is almost perfect for small values of $U$, with increasing deviations up to a few percent for larger $U$. However, at $U=$ 3, the DQMC results for the first Matsubara frequency differ considerably from those of the $\mathrm{fRG}^{*}$ and the PA. Moreover, comparing the results for the first and second Matsubara frequencies at $\mathbf{k}=(\pi, 0)$ [last data points in Figs. 13(b) and 13(d)] reveals a discrepancy with an important physical implication: The onset of the pseudogap opening [87-89], resulting from quasi-identical values for the first two Matsubara frequencies at $\mathbf{k}=(\pi, 0)$, is observed at $U=3$ in the DQMC but not (yet) in the fRG* and the PA. The reason is the neglect of fully two-particle irreducible diagrams. In the fRG* and the PA, the absolute value at the first Matsubara frequency is $11 \%$ smaller than at the second one and the gap opening sets in only for larger interactions (not shown). 

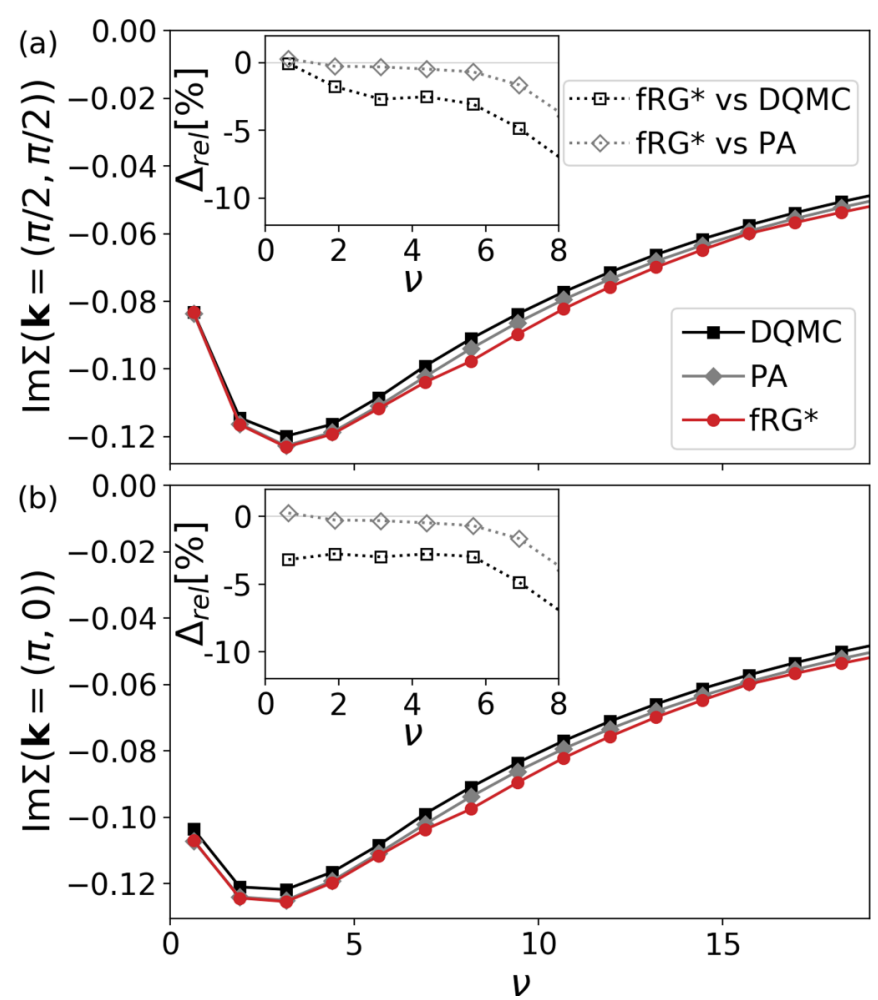

FIG. 14. Imaginary part of the self-energy at (a) the nodal and (b) the antinodal point, as obtained by the fRG* (red), the PA (gray), and the DQMC (black), for $U=2$ and $1 / T=5$. The inset shows the relative difference of the $\mathrm{fRG}^{*}$ with respect to the PA (gray) and DQMC (black).

We also compare the behavior of the self-energy as a function of frequency in Fig. 14 for a representative value of $U=2$ (and $1 / T=5$ ). At small frequencies the self-energy shows typical Fermi-liquid behavior $\operatorname{Im} \Sigma(i v \rightarrow 0) \rightarrow 0$ at both the nodal and antinodal points. The antinodal point is affected more strongly by correlation effects, with an increased absolute value for the lowest Matsubara frequencies. In general, both Figs. 14(a) and 14(b) indicate that, for these parameters, the resulting self-energy does not develop a momentum-selective gap. In Fig. 14 the fRG* and the PA exhibit larger deviations from each other in the intermediateto high-frequency range, although the $\mathrm{fRG}^{*}$ perfectly fulfills the postprocessing SDE (see also Fig. 21 in Appendix D). The differences are due to the specific implementation of the high-frequency asymptotics of the two-particle vertex [69]: In the $\mathrm{fRG}^{*}$ the asymptotic functions are calculated and stored explicitly [57], retaining a smaller tensor for the low-frequency range compared to the PA, where a large tensor over many fermionic and bosonic frequencies is used and the values outside are constructed from the ones at the edges [75]. The former is numerically more efficient but has the drawback of kinks arising at the transition between the different tensors (see Fig. 14). On a quantitative level, in the full Green's function $G(\mathbf{k}, i v)$ the differences between the frequency dependence of the $\mathrm{fRG}^{*}$ and the PA self-energy are almost negligible due to the large $i v$ contribution of the bare Green's function $G_{0}(\mathbf{k}, i v)$ [see Eq. (2)]. We verified that for smaller interactions (and also larger low-frequency tensors)
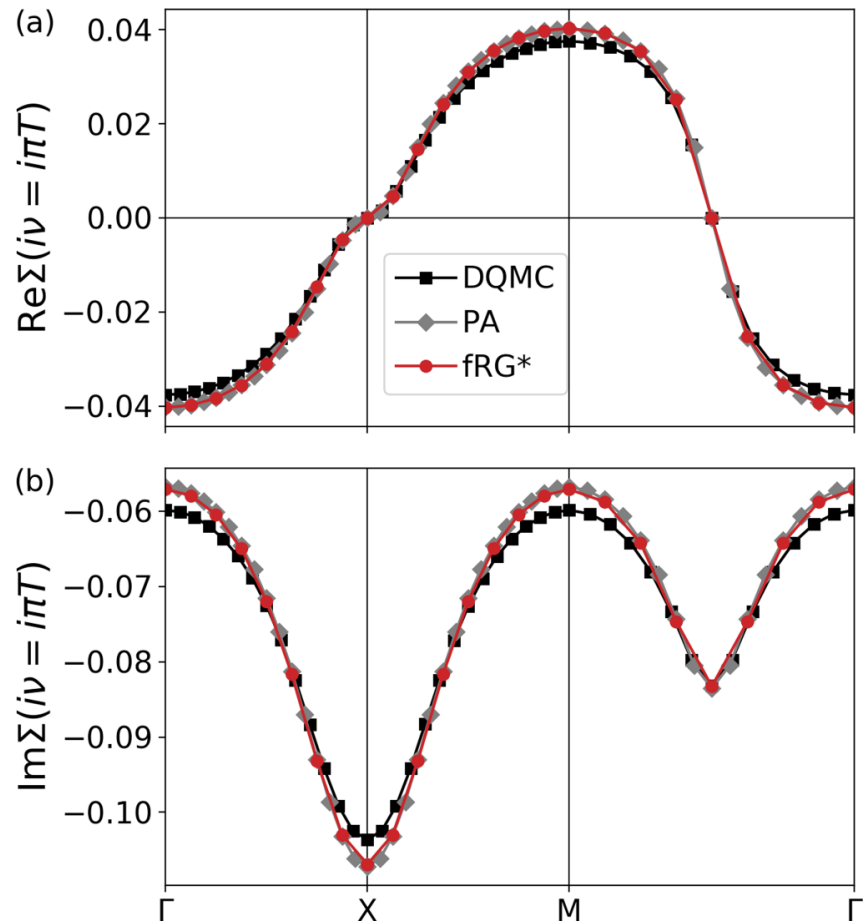

FIG. 15. (a) Real and (b) imaginary parts of the self-energy, as obtained by the fRG* (red), the PA (gray), and the DQMC (black), for $U=2$ and $1 / T=5$.

excellent convergence in frequencies, momenta, and loops can be achieved [70].

Finally, a comparison of the self-energy as a function of momentum for the same representative parameters of $U=2$ and $1 / T=5$ is performed in Fig. 15. We find that the $\mathrm{fRG}^{*}$ (red) perfectly reproduces the PA (gray) solution. Concerning their agreement with the DQMC (black), we observe a difference of $3 \%$ at $X$ and perfect agreement at the nodal point (between $M$ and $\Gamma$ ). The differences at momenta far away from the Fermi surface have little influence since it is the Green's function and not the self-energy that directly enters the calculation of observables.

\section{Double occupancy}

Finally, we determine the interaction dependence of the double occupancy. The results of the different methods for $1 / T=5$ are shown in Fig. 16. In the fRG*, the double occupancy is obtained through the postprocessed susceptibilities using Eq. (8); Eq. (9) is used by the PA. Both expressions are equivalent by virtue of the SDE (11). In the DQMC the Hubbard-Stratonovich transformation yields, for each configuration, an effectively noninteracting system. Then Wick's theorem applies and the double occupancy can be directly measured from the static single-particle Green's function. The comparison between the $\mathrm{RRG}^{*}$ and PA as well as with the DQMC shows good agreement and reflects the behavior observed for the involved susceptibilities, as already discussed in Sec. V A. 


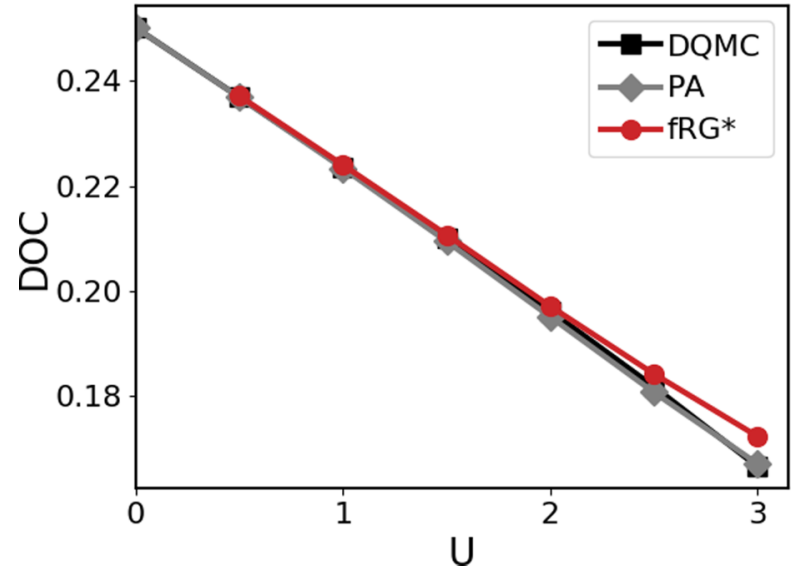

FIG. 16. Double occupancy (DOC) as a function of $U$, as obtained by the $\mathrm{fRG}^{*}$ (red), the PA (gray), and the DQMC (black) for $1 / T=5$.

\section{RESULTS AWAY FROM HALF FILLING}

In the presence of finite doping and additional next-nearestneighbor hopping $t^{\prime}$, the physical behavior is much richer and not exclusively driven by AF fluctuations anymore. Since we expect the superconducting $d$-wave component of the two-particle vertex to become more important here, we also include the $d$-wave form factor.

In the following we present the fRG* results for the evolution of the different susceptibilities away from half filling, together with their comparison to the PA and DQMC data. Specifically, we consider the parameters $t^{\prime}=-0.2$ for the next-nearest-neighbor hopping and $\mu=-0.35,-0.7,-1.4$, and -2 for the chemical potential. Due to the self-energy flow in the $\mathrm{RRG}^{*}$ the initial chemical potential is renormalized, leading to a different filling at the end of the flow. This effect is very small close to half filling and increases with the doping $\delta=1-\langle\hat{n}\rangle$; see Fig. 17 where the magnetic susceptibility $\chi_{\mathrm{M}}(\mathbf{q}, i \omega=0)$ in momentum space is shown for $U=2$ and $1 / T=5$. In Fig. 18 we report the compressibility $\kappa$, the charge density wave $\chi_{\mathrm{CDW}}$, and the superconducting $\chi_{\mathrm{SC}}(s-$ and $d$-wave) susceptibility as a function of doping, for the same parameters. We note that here $\chi_{\mathrm{CDW}}$ and $\chi_{\mathrm{SC}, s}$ are not equivalent anymore.

The magnetic susceptibility dominates for small dopings. It is maximal at the commensurate $\mathrm{AF}$ wave vector $(\pi, \pi)$ for $\mu=-0.35$ [Fig. 17(a)] and $\mu=-0.7$ [Fig. 17(b)] and at incommensurate wave vectors for larger values of the doping [Figs. 17(c) and 17(d)], consistent with previous fRG findings $[59,90]$. In particular, we do not find a pairing instability for any doping at a temperature as high as $1 / T=5$. At the same time, with increasing $\delta$ the $d$-wave pairing eventually overcomes the tendency towards magnetic ordering, i.e., the maximum of the (in)commensurate peak in $\chi_{M}(\mathbf{q}, i \omega=0)$ is lower than $\chi_{\mathrm{SC}, d}$. We note that while the AF susceptibility gradually evolves from the beginning of the flow $[9,48,59,91]$, the superconducting $d$-wave susceptibility emerges only in proximity to the critical scale. This indicates that the AF fluctuations are responsible for the $d$-wave pairing. The parameter regime presented here is far away from any instability. Hence, for a finite doping we expect the $d$-wave pairing susceptibility

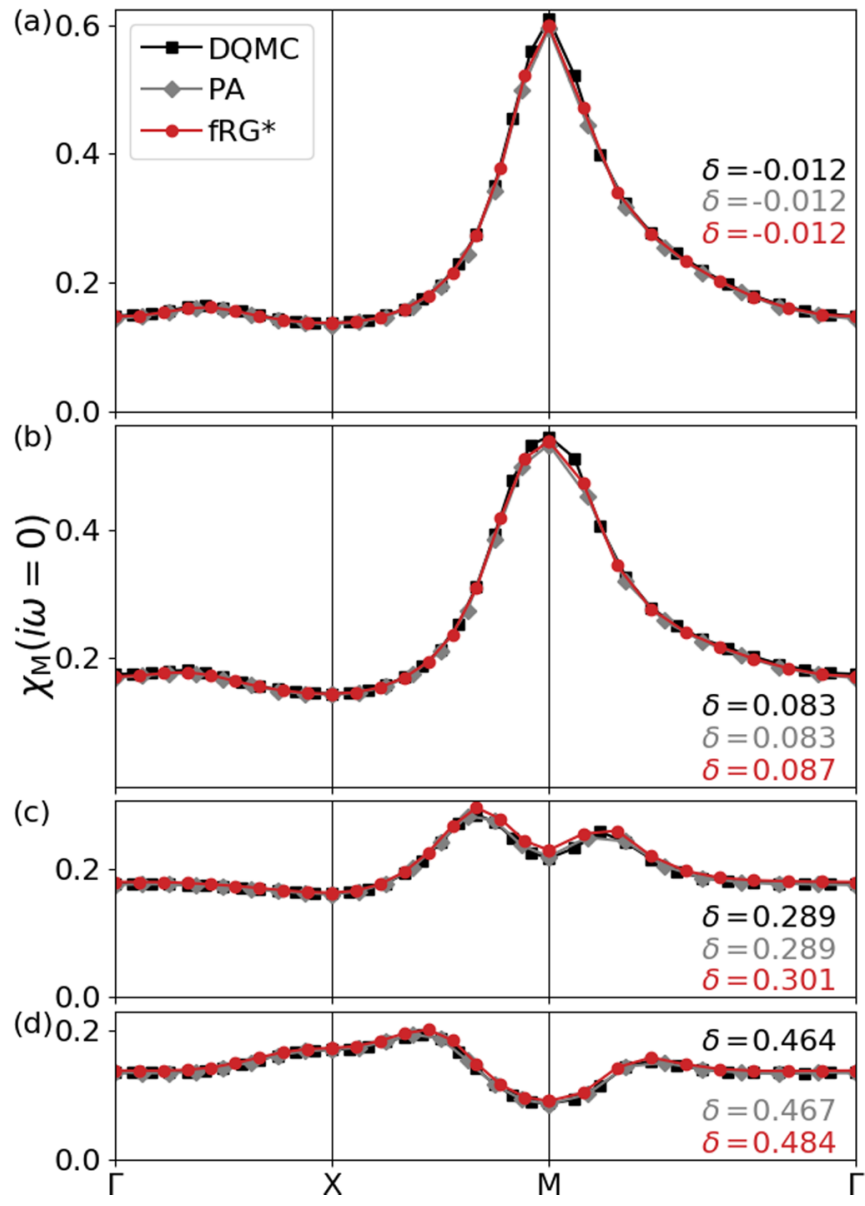

FIG. 17. Magnetic susceptibility $\chi_{\mathrm{M}}(i \omega=0)$ as obtained by the $\mathrm{fRG}^{*}$ (red), the PA (gray), and the DQMC (black) for $U=2, t^{\prime}=$ $-0.2,1 / T=5$, and different values of the doping resulting from (a) $\mu=-0.35$, (b) $\mu=-0.7$, (c) $\mu=-1.4$, and (d) $\mu=-2$.

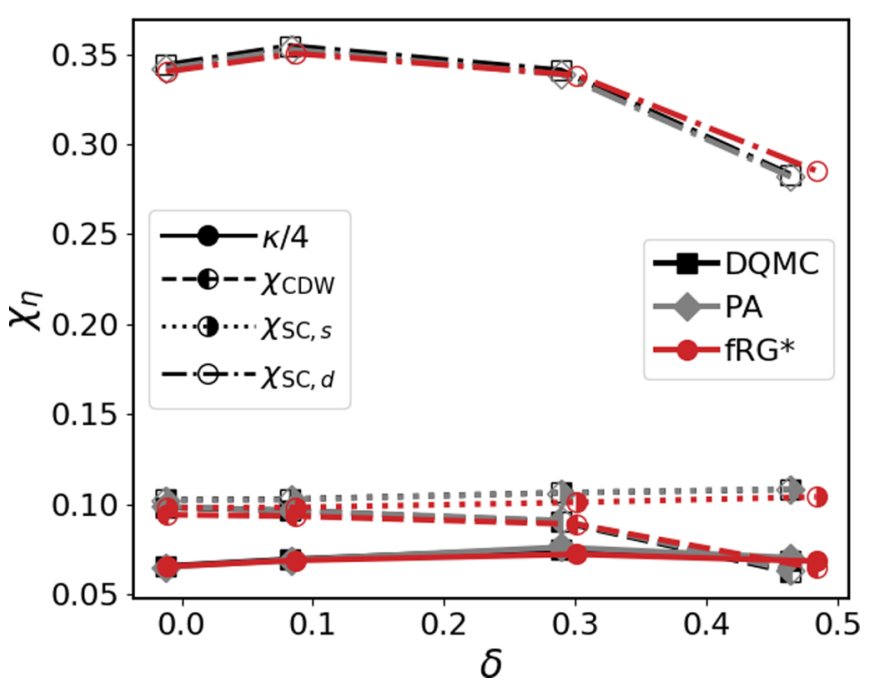

FIG. 18. Compressibility $\kappa$, charge density wave $\chi_{\mathrm{CDW}}$, and superconducting susceptibility $\chi_{\mathrm{SC}}$ in $s$ and $d$ waves as obtained by the $\mathrm{fRG}^{*}$ (red), the PA (gray), and the DQMC (black) for $U=2$, $1 / T=5$, and different values of the doping $\delta$. 
to increase only at lower temperatures. Due to the high computational cost of low- $T$ calculations (specifically to be able to accurately parametrize the frequency dependence of the twoparticle vertex), we cannot access the superconducting transition temperature at the moment. For the temperatures considered here, the onset of a large $d$-wave pairing interaction is likely a high-temperature precursor of a superconducting phase at lower temperature: As the temperature is further decreased, the relevance of the $d$-wave pairing should increase.

The agreement of the $\mathrm{RRG}^{*}$ with the PA and the numerically exact DQMC data is very good also away from half filling. We refrain here from providing relative differences because the data at fixed $\mu$ correspond to different fillings. Moreover, due to the high numerical cost, the present calculation including $s$ - and $d$-wave form factors is not fully converged in frequencies. This hardly affects the susceptibilities, while the quantitative accuracy of the self-energy appears to be more sensitive.

\section{CONCLUSION AND OUTLOOK}

In this work we illustrated how it is possible to achieve, by means of the fRG, a quantitatively accurate description of correlated electrons on 2D lattices, by implementing proper enhancements to the conventional algorithms. Our starting point was the significant progress recently obtained in [57], which combined the truncated unity fRG [48], a clever frequency representation [21,69], and the multiloop extension of the approach described in Refs. $[44,45]$. While the latter advances suffice for impurity models [44,46], the missing piece for a quantitatively accurate description of the electronic correlations in two dimensions is to make the self-energy flow consistent with the truncated unity scheme. Specifically, we showed that replacing the corresponding flow equation by the direct derivative of the Schwinger-Dyson equation allows us to sum up the contributions of the different channels in the correct proportion. The postprocessed computation from the propagator and interaction vertex at the end of the flow exactly fulfills the SDE. As a consequence, the resulting self-energy is independent of the chosen cutoff scheme.

This methodological improvement is needed for converging, at a high degree of numerical accuracy, the multiloop fRG results to the PA and for obtaining quantitatively reliable fRG data for the 2D Hubbard model at half filling as well as upon hole doping. In particular, by comparing the converged fRG data to the PA and DQMC, satisfactory agreement between the corresponding values of the self-energies and physical response functions could be established up to intermediate interaction strength. We stress that such quantitative agreement for the 2D Hubbard model cannot be obtained by exploiting conventional (e.g., one-loop-based) truncations of the fRG flow. Minor deviations between the fRG and PA (on one side) and DQMC (on the other side) are instead observed, as expected, by increasing the interaction values. Further optimization and parallelization of the code [54] will allow us to overcome the present restriction to essentially a single $s$-wave form factor. This is necessary to explore broader parameter regions. Also qualitatively, the presented Schwinger-Dyson form of the self-energy flow equation turns out to be essential in order to capture the pseudogap opening in the 2D Hubbard model at half filling [92].

The presented advancements of fRG-based computation schemes constitute the basis for its extensions to more general systems and for its combination $[62,63]$ with nonperturbative many-body methods, such as DMFT. Note that also for $a b$ initio investigations, the consistent summation of different scattering channels is of clear importance [93] and the fRG might prove useful in this regard as well. Hence, our study establishes a promising route towards quantitative fRG analyses of electronic phase diagrams at all coupling strengths and for an fRG-based investigation of emerging energy scales, competing instabilities, and response functions in wider classes of quantum materials of high relevance for cutting-edge condensed matter research.

\section{ACKNOWLEDGMENTS}

The authors thank P. Chalupa, J. von Delft, J. Ehrlich, S. Heinzelmann, K. Held, M. Klett, T. A. Maier, W. Metzner, D. Rohe, D. J. Scalapino, T. Schäfer, A. Tagliavini, D. Vilardi, and N. Wentzell for valuable discussions and A. Lebedev for his support with the computing infrastructure. We acknowledge financial support from the Deutsche Forschungsgemeinschaft (DFG) through ZUK 63 and Projects No. AN 815/4-1 and No. AN 815/6-1, through DFG-RTG "Quantum Many-Body Methods in Condensed Matter Systems", through Germany's Excellence Strategy-EXC-2111-390814868 (F.B.K.), and from Austrian Science Fund (FWF) through Project No. I 2794-N35 (A.T.). Calculations were done in part on the Vienna Scientific Cluster. The authors also gratefully acknowledge the computing time granted through JARA on the supercomputer JURECA at Forschungszentrum Jülich [94].

\section{APPENDIX A: FLOWING VS POSTPROCESSED SUSCEPTIBILITIES}

Typical response functions, such as susceptibilities, can be calculated from the one-particle Green's function and the twoparticle vertex at the end of the flow. In this postprocessing scheme, a susceptibility is obtained by integrating over the energies and momenta of Green's functions attached to the external legs of the vertex. Alternatively, one can set up additional fRG flow equations for the response functions, with the typical structure of a single-scale propagator connecting higher-order vertices [64]. For simplified fRG schemes, the latter approach is often preferential (for an example see the calculation of the density profile near a static impurity in a Luttinger liquid [33]). One reason is that the single-scale propagator restricts internal integrations to the flowing energy scale $\Lambda$. Performing such integrations does not require an accurate description of the vertices at all energies, but just at the current relevant scale.

In (converged) multiloop fRG, the ambiguity in the computation of response functions is resolved and both schemes become equivalent [57,67]. Importantly, such quantitative fRG schemes require an accurate parametrization for all frequencies since, in the multiloop corrections (and already when including the Katanin correction [65] to the one-loop 


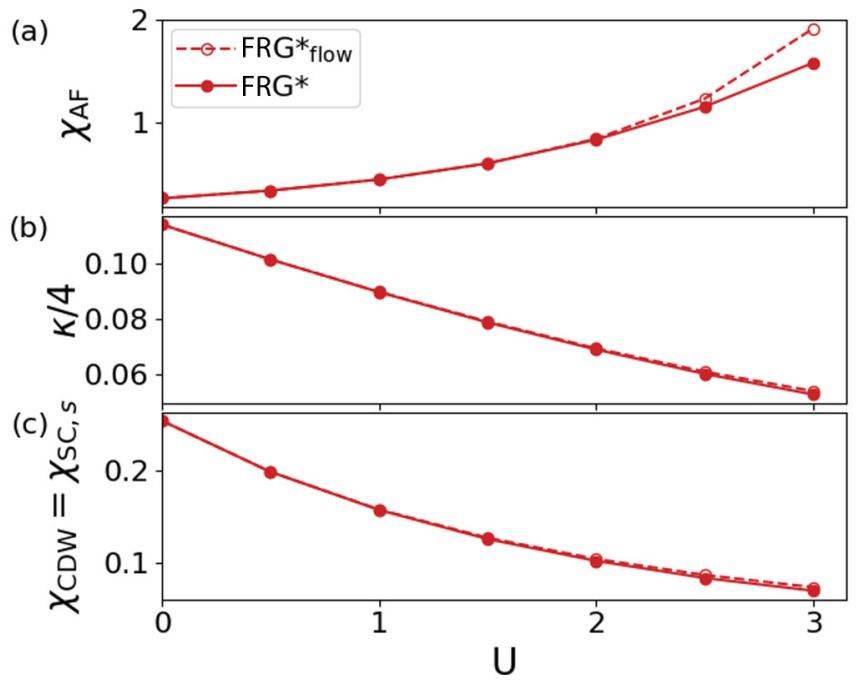

FIG. 19. (a) Antiferromagnetic susceptibility $\chi_{\mathrm{AF}}$, (b) compressibility $\kappa$, and (c) charge density wave $\chi_{\mathrm{CDW}}=\chi_{\mathrm{SC}, s}$ as a function of $U$ obtained by the $\mathrm{fRG}^{*}$ for $1 / T=5$ and half filling. Postprocessed (closed symbols) and flowing results (open symbols) are shown.

scheme), there is no single-scale propagator restricting the loop integration. Similarly, the postprocessed susceptibilities involve integrations over all arguments of the vertex.

While flowing susceptibilities may be more convenient in simplified fRG implementations, given the high resolution of the two-particle vertex in our study, we can also compute postprocessed susceptibilities to high accuracy. In fact, there are reasons why the postprocessed susceptibilities are more accurate in such a quantitative fRG approach. On the one hand, the multiloop corrections affect the flowing susceptibilities already at second order in the (renormalized) interaction (see Fig. 2 of Ref. [57] or Fig. 8 of Ref. [67]); however, they affect the two-particle vertex, and thus the postprocessed susceptibilities, starting at third order (see Fig. 2 of Ref. [67]). Accordingly, the latter have been found to converge faster in the number of loops [57]. On the other hand, the postprocessed local, equal-spin charge susceptibility obeys its sum rule exactly, using a vertex computed at any loop order. By contrast, without full loop convergence, the flowing susceptibility does not [46]. The postprocessing scheme thus has a closer connection to the exact relations from which the multiloop equations are derived. Hence, one can expect better agreement with the PA (i.e., faster convergence in loop order), as it has been generally observed for the susceptibilities as well as for a postprocessed self-energy (by using the SDE) $[46,57,70]$. For this reason we here use the postprocessed susceptibilities; a comparison to the ones obtained from the flow of the response functions is shown in Fig. 19. It provides an indication of the $\mathrm{fRG}^{*}$ convergence with respect to momenta, frequencies, and loop numbers. The agreement is within numerical accuracy for almost all data points. The only exception is $\chi_{\mathrm{AF}}$, where for $U>2.5$ it is difficult to converge the $\mathrm{fRG}^{*}$ calculations in frequencies and loop numbers. For $U=3$, the AF susceptibility shown in Fig. 8 is not converged yet in frequencies and loop numbers. For a more detailed comparison of the flowing results we refer to Ref. [70].
TABLE I. fRG* parameters used in Sec. V. The additional 24 bosonic patching points in $N_{\mathbf{q}}$ are distributed around $\mathbf{k}=(\pi, \pi)$. Here $N_{\mathbf{k}}$ is the number of points in the momentum integration of the fermionic bubble. The frequency ranges of the vertex and vertex asymptotics are proportional to the number of positive fermionic frequencies $N_{f^{+}}$of the low-frequency object with three dependences. Due to computational limits, the calculations for $U>2$ are not converged with respect to the number of loops $N_{\ell}$ and self-energy iterations $N_{\Sigma \text {-iter }}$.

\begin{tabular}{cccccc}
\hline \hline$U$ & $N_{\mathbf{q}}$ & $N_{\mathbf{k}}$ & $N_{f^{+}}$ & $N_{\ell}$ & $N_{\Sigma \text {-iter }}$ \\
\hline 0.0 & $12 \times 12+24$ & $60 \times 60$ & 4 & 1 & 1 \\
0.5 & $12 \times 12+24$ & $60 \times 60$ & 4 & 16 & 5 \\
1.0 & $16 \times 16+24$ & $80 \times 80$ & 4 & 16 & 5 \\
1.5 & $16 \times 16+24$ & $80 \times 80$ & 4 & 16 & 5 \\
2.0 & $16 \times 16+24$ & $80 \times 80$ & 6 & 26 & 5 \\
2.5 & $16 \times 16+24$ & $80 \times 80$ & 6 & 28 & 5 \\
3.0 & $16 \times 16+24$ & $80 \times 80$ & 6 & 28 & 5 \\
\hline \hline
\end{tabular}

\section{APPENDIX B: TECHNICAL PARAMETERS OF fRG*}

We report the technical parameters for the results of Secs. V and VI in Tables I and II, respectively.

\section{APPENDIX C: IMPLEMENTATION OF THE SELF-ENERGY FLOW}

In the fRG* approach we replace the conventional flow equation of the self-energy together with its multiloop corrections by

$$
\begin{aligned}
\dot{\Sigma}(\mathbf{k}, i v)= & \dot{\Sigma}_{G G G}(\mathbf{k}, i v) \\
& +\dot{\Sigma}_{p h}(\mathbf{k}, i v)+\dot{\Sigma}_{\overline{p h}}(\mathbf{k}, i v)+\dot{\Sigma}_{p p}(\mathbf{k}, i v) .
\end{aligned}
$$

Recall that the Hartree part is implicitly taken into account by shifting the chemical potential by $U\left\langle\hat{n}_{\sigma}\right\rangle$ (with $\mu=0$ for half filling). The first part does not depend on the full vertex,

$$
\begin{aligned}
\dot{\Sigma}_{G G G}(\mathbf{k}, i v)= & -U^{2} \sum_{\mathbf{k}^{\prime} \mathbf{q}} \sum_{i v^{\prime} i \omega}\left[\dot{G}^{\Lambda}\left(\mathbf{k}^{\prime}, i v^{\prime}\right) G^{\Lambda}\left(\mathbf{k}^{\prime}+\mathbf{q}, i v^{\prime}+i \omega\right)\right. \\
& \times G^{\Lambda}(\mathbf{k}+\mathbf{q}, i v+i \omega) \\
& +G^{\Lambda}\left(\mathbf{k}^{\prime}, i v^{\prime}\right) \dot{G}^{\Lambda}\left(\mathbf{k}^{\prime}+\mathbf{q}, i v^{\prime}+i \omega\right) \\
& \times G^{\Lambda}(\mathbf{k}+\mathbf{q}, i v+i \omega) \\
& +G^{\Lambda}\left(\mathbf{k}^{\prime}, i v^{\prime}\right) G^{\Lambda}\left(\mathbf{k}^{\prime}+\mathbf{q}, i v^{\prime}+i \omega\right) \\
& \left.\times \dot{G}^{\Lambda}(\mathbf{k}+\mathbf{q}, i v+i \omega)\right], \quad(\mathbf{C} 2)
\end{aligned}
$$

TABLE II. fRG* parameters used in Sec. VI. The calculations are converged with respect to $N_{\mathbf{q}}, N_{\mathbf{k}}, N_{\ell}$, and $N_{\Sigma \text {-iter. }}$ As the calculations with an additional $d$-wave form factor are numerically challenging, the frequency range was fixed to $N_{f^{+}}=2$.

\begin{tabular}{lccccc}
\hline \hline$\delta$ & $N_{\mathbf{q}}$ & $N_{\mathbf{k}}$ & $N_{f^{+}}$ & $N_{\ell}$ & $N_{\Sigma \text {-iter }}$ \\
\hline-0.012 & $18 \times 18$ & $90 \times 90$ & 2 & 22 & 5 \\
0.087 & $18 \times 18$ & $90 \times 90$ & 2 & 26 & 5 \\
0.301 & $18 \times 18$ & $90 \times 90$ & 2 & 26 & 5 \\
0.484 & $18 \times 18$ & $90 \times 90$ & 2 & 26 & 5 \\
\hline \hline
\end{tabular}


and reproduces second-order perturbation theory with renormalized propagators. Applying the convolution theorem twice, it can be calculated efficiently by using fast-Fourier-transform routines $\mathcal{F}$ in real space:

$$
\begin{aligned}
\dot{\Sigma}_{G G G}(\mathbf{k}, i v)= & -U^{2} \mathcal{F}\left[\sum_{i v^{\prime} i \omega} \dot{G}^{\Lambda}\left(\mathbf{R}, i v^{\prime}\right) G^{\Lambda}\left(-\mathbf{R}, i v^{\prime}+i \omega\right) G^{\Lambda}(\mathbf{R}, i v+i \omega)+G^{\Lambda}\left(\mathbf{R}, i v^{\prime}\right) \dot{G}^{\Lambda}\left(-\mathbf{R}, i v^{\prime}+i \omega\right) G^{\Lambda}(\mathbf{R}, i v+i \omega)\right. \\
& \left.+G^{\Lambda}\left(\mathbf{R}, i v^{\prime}\right) G^{\Lambda}\left(-\mathbf{R}, i v^{\prime}+i \omega\right) \dot{G}^{\Lambda}(\mathbf{R}, i v+i \omega)\right](\mathbf{k}) .
\end{aligned}
$$

Each of the vertex-dependent contributions to the self-energy flow (C1), $\dot{\Sigma}_{p h}(k, i v), \dot{\Sigma}_{\overline{p h}}(k, i v)$, and $\dot{\Sigma}_{p p}(k, i v)$ in the respective channels, contains similar terms regarding the $\Lambda$ derivative: Applying the product rule, we obtain the derivative of the two-particle reducible vertex, of the propagator bubble, and of the propagator closing the external loop. As the full expression of the self-energy flow equation can be easily derived from the SDE (12), we show here only the $p h$ contribution explicitly,

$$
\begin{aligned}
\dot{\Sigma}_{p h}(\mathbf{k}, i v)= & \sum_{\mathbf{q} i \omega} \sum_{m} f_{m}^{*}(\mathbf{k})\left[\sum_{i v^{\prime}} \sum_{n} \dot{\Phi}_{p h, m n}^{\Lambda}\left(\mathbf{q}, i \omega, i v, i v^{\prime}\right) \Pi_{p h, n 0}^{\Lambda}\left(\mathbf{q}, i \omega, i v^{\prime}\right) 4 \pi^{2} U f_{0}(\mathbf{k})\right] G^{\Lambda}(\mathbf{k}+\mathbf{q}, i v+i \omega) \\
& +\sum_{\mathbf{q} i \omega} \sum_{m} f_{m}^{*}(\mathbf{k})\left[\sum_{i \nu^{\prime}} \sum_{n} \Phi_{p h, m n}^{\Lambda}\left(\mathbf{q}, i \omega, i v, i v^{\prime}\right) \dot{\Pi}_{p h, n 0}^{\Lambda}\left(\mathbf{q}, i \omega, i v^{\prime}\right) 4 \pi^{2} U f_{0}(\mathbf{k})\right] G^{\Lambda}(\mathbf{k}+\mathbf{q}, i v+i \omega) \\
& +\sum_{\mathbf{q} i \omega} \sum_{m} f_{m}^{*}(\mathbf{k})\left[\sum_{i v^{\prime}} \sum_{n} \Phi_{p h, m n}^{\Lambda}\left(\mathbf{q}, i \omega, i v, i v^{\prime}\right) \Pi_{p h, n 0}^{\Lambda}\left(\mathbf{q}, i \omega, i v^{\prime}\right) 4 \pi^{2} U f_{0}(\mathbf{k})\right] \dot{G}^{\Lambda}(\mathbf{k}+\mathbf{q}, i v+i \omega),
\end{aligned}
$$

where $V_{\Lambda=0, s s}=4 \pi^{2} U$ due to the normalization of the form factors [57]. The three parts are similar in structure and can be calculated using the same procedure, merely exchanging the vertex or the propagator at a specific scale with its derivative at this scale. In practice, the bubble $\Pi_{p h, m n}^{\Lambda}(\mathbf{q}, i \omega, i v)$ and its derivative are already calculated for the conventional $1 \ell$ and $2 \ell$ vertex flows. The multiplications inside the square brackets are of the same form as the ones on the rhs of the flow equations for the vertex, where to the right of the bubble only the bare vertex is inserted. The resulting effective vertices are projected into the purely fermionic notation by the form factors $f_{m}^{*}(\mathbf{k})$ and $f_{0}(\mathbf{k})$ and then contracted with a propagator (or scale derivative of a propagator) as in the conventional $1 \ell$ flow equation for the self-energy. Hence, only the computation of $\dot{\Sigma}_{G G G}(\mathbf{k}, i v)$ has to be implemented separately.

Both the fRG* and the conventional multiloop corrections of the self-energy flow can be calculated fully only after the rhs of the vertex, as they depend on the scale derivative of the vertex. The resulting derivative of the self-energy, which affects the Katanin substitution of the single-scale propagator, must then be used for another evaluation of the vertex flow. This is iterated until the change in $\dot{\Sigma}$ is negligible. Note that, in contrast to the conventional $1 \ell$ flow equation for the self-energy, the self-energy flow equation in SchwingerDyson form depends explicitly on $\dot{G}$ and therefore also on $\dot{\Sigma}$. Therefore, we use the conventional $1 \ell$ flow as the first estimate of $\dot{\Sigma}$. The results obtained by retaining only this part of $\dot{\Sigma}$ are labeled as "no $\Sigma$-iter" in Figs. 6, 7, and 20 and are compared to the fully converged ones.

\section{APPENDIX D: FURTHER SELF-ENERGY RESULTS}

In addition to the results presented in Sec. III D, we illustrate in Fig. 20 the effect of the self-energy iterations on the momentum dependence of the self-energy at $i \nu=i \pi T$ for both the fRG and fRG*. This supplements the results for the frequency dependence for $U=2$ and $1 / T=5$ shown in Fig. 6. Consistent with the observation there, the self-energy iterations lead to small corrections in the fRG*, while in the conventional fRG flow the effect on the lowest Matsubara frequency is almost negligible.
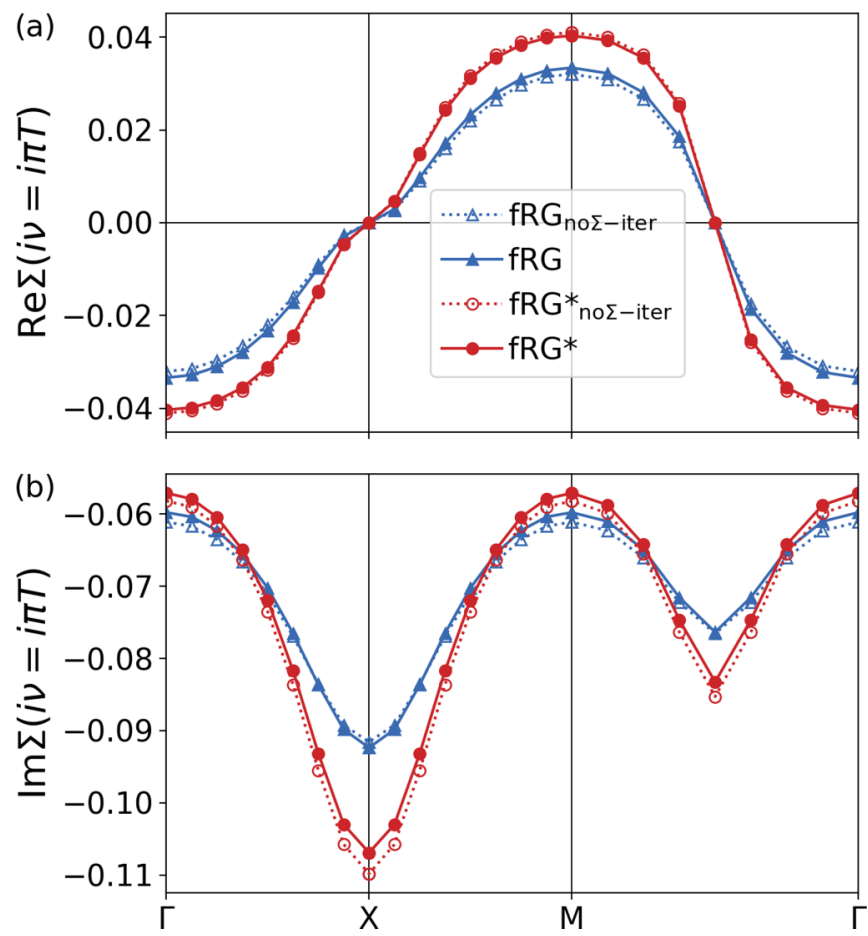

FIG. 20. (a) Real and (b) imaginary parts of the self-energy as obtained by the conventional fRG (blue) and fRG* (red), with and without self-energy iteration, respectively, for $U=2$ and $1 / T=5$ (at half filling). 

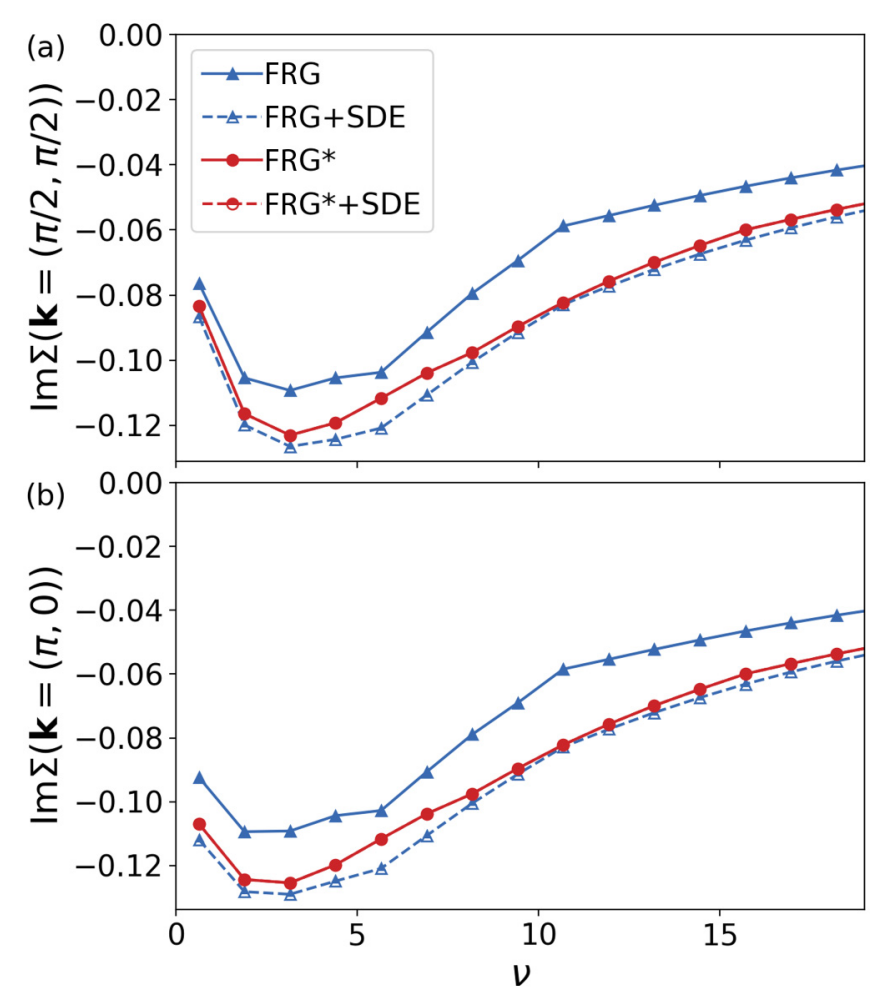

FIG. 21. Imaginary part of the self-energy at (a) the nodal and (b) the antinodal point, as obtained by fRG (blue) and fRG* flow (red), together with the respective postprocessed results (dashed lines), for $U=2$ and $1 / T=5$ (at half filling). Within the $\mathrm{fRG}^{*}$, the postprocessed results (red dashed lines) lie exactly on top of the fRG* flow results (red solid lines).

Furthermore, in Fig. 21 we present the postprocessed results for the self-energy as a function of frequency, in analogy to the ones for the momentum dependence shown in Fig. 5. Also here the fRG*, which accounts for the form-factor projections in the different channels, yields perfect agreement between the flowing and the postprocessed results for the whole frequency range, unlike the conventional fRG scheme.

\section{APPENDIX E: EQUIVALENCE $\chi_{S C}(q=0)=\chi_{D}(q=(\pi, \pi))$ AT HALF FILLING}

The proof of equivalence between $\chi_{\mathrm{SC}}$ and $\chi_{\mathrm{CDW}}$ at half filling traced in Ref. [95] is elaborated in the following. The Hubbard model (1) is SU(2) spin symmetric. At both half filling and with only nearest-neighbor hopping it is furthermore SU(2) charge symmetric:

$$
\hat{\mathcal{H}}=-t \sum_{\langle i j\rangle \sigma}\left(\hat{c}_{i \sigma}^{\dagger} \hat{c}_{j \sigma}+\text { H.c. }\right)+U \sum_{i}\left(\hat{n}_{i \uparrow} \hat{n}_{i \downarrow}-\frac{\hat{n}_{i \uparrow}+\hat{n}_{i \downarrow}}{2}\right) \text {. }
$$

This charge symmetry implies a degeneracy between the local $s$-wave spin-singlet pairing and the charge density wave chan- nel $\chi_{\mathrm{SC}}(\mathbf{q}=\mathbf{0})=\chi_{\mathrm{D}}(\mathbf{q}=(\pi, \pi))$, which we also observe in the numerical results presented in Sec. V.

In order to prove the charge $\mathrm{SU}(2)$ symmetry for the Hamiltonian (E1), we introduce the spinor operators

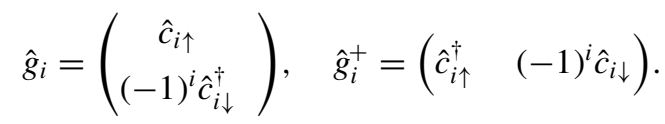

The Hamiltonian (E1) then reads

$$
\hat{\mathcal{H}}=-t \sum_{\langle i j\rangle}\left(\hat{g}_{i}^{\dagger} \hat{g}_{j}+\text { H.c. }\right)-\frac{U}{2} \sum_{i}\left(\hat{P}_{i}^{\dagger} \hat{P}_{i}+\hat{P}_{i} \hat{P}_{i}^{\dagger}\right),
$$

where $\hat{P}_{i}$ and $\hat{P}_{i}^{\dagger}$ are given by

$$
\begin{aligned}
& \hat{P}_{i}=\frac{1}{2}(-1)^{i} \hat{g}_{i}^{\dagger}\left(i \tau^{2}\right)\left(\hat{g}_{i}^{\dagger}\right)^{\mathrm{T}}=\hat{c}_{i \uparrow}^{\dagger} \hat{c}_{i \downarrow}, \\
& \hat{P}_{i}^{\dagger}=\frac{1}{2}(-1)^{i}\left(\hat{g}_{i}\right)^{\mathrm{T}}\left(-i \tau^{2}\right) \hat{g}_{i}=\hat{c}_{i \downarrow}^{\dagger} \hat{c}_{i \uparrow},
\end{aligned}
$$

with $\tau^{2}$ the second Pauli matrix. Performing a global SU(2) transformation for the spinor operators $\hat{g}_{i} \rightarrow U^{\dagger} \hat{g}_{i}$ and $\hat{g}_{i}^{\dagger} \rightarrow$ $\hat{g}_{i}^{\dagger} U$, where $U \in \mathrm{SU}(2)$ is a $2 \times 2$ matrix which does not depend on the lattice site, we can show that the Hamiltonian (E3) is invariant under this transformation. For the first hopping term, it is clear that $\left(\hat{g}_{i}^{\dagger} \hat{g}_{j}+\right.$ H.c. $)$ is invariant under this transformation. For the interaction term, we consider the operator $\hat{P}_{i}$ under the above $\mathrm{SU}(2)$ transformation

$$
\hat{P}_{i} \stackrel{\mathrm{SU}(2)}{\longrightarrow} \frac{1}{2}(-1)^{i} \hat{g}_{i}^{\dagger} U\left(i \tau^{2}\right) U^{\mathrm{T}}\left(\hat{g}_{i}^{\dagger}\right)^{\mathrm{T}},
$$

and applying the property $\operatorname{det}(U)=1$ of the $2 \times 2 \mathrm{SU}(2)$ matrix, we can actually prove that $U\left(i \tau^{2}\right) U^{\mathrm{T}}=i \tau^{2}$. This means that under the above transformation, the operator $\hat{P}_{i}$ is invariant and so is the $\hat{P}_{i}^{\dagger}$ operator. Thus, the total Hamiltonian (E3) is invariant under the SU(2) transformation.

Like for the spin symmetry, the representation of the density operator $\hat{\mathbf{N}}_{i}$ in terms of the $\mathrm{SU}(2) \simeq \mathrm{SO}(3) \times Z_{2}$ symmetry group generators is $\hat{\mathbf{N}}_{i}=\frac{1}{2}(-1)^{i} \hat{g}_{i}^{\dagger}\left(\tau^{1}, \tau^{2}, \tau^{3}\right) \hat{g}_{i}$. The three components of $\hat{\mathbf{N}}_{i}$ are invariant under $\mathrm{SO}(3)$ rotations. Furthermore, we can express them as $\hat{\mathbf{N}}_{i}=\left(\operatorname{Re} \hat{\Delta}_{i}, \operatorname{Im} \hat{\Delta}_{i}, \hat{D}_{i}\right)$, with $\hat{\Delta}_{i}=\hat{c}_{i \uparrow}^{\dagger} \hat{c}_{i \downarrow}^{\dagger}$ and $\hat{D}_{i}=\frac{1}{2}(-1)^{i}\left(\hat{n}_{i \uparrow}+\hat{n}_{i \downarrow}-1\right)$, which explicitly shows the equivalence of the correlation functions $\left\langle\left(\operatorname{Re} \hat{\Delta}_{i}\right)\left(\operatorname{Re} \hat{\Delta}_{j}\right)\right\rangle=\left\langle\hat{D}_{i} \hat{D}_{j}\right\rangle$.

In the main text, the density susceptibility $\chi_{\mathrm{D}}(\mathbf{q})$ of Eq. (6) was defined by using $(-1)^{i} \hat{D}_{i}$ and the superconducting one $\chi_{\mathrm{SC}}(\mathbf{q})$ of Eq. (7) by using $\operatorname{Re} \hat{\Delta}_{i}$. Thus, as a result of the charge SU(2) symmetry as described above, the susceptibilities are related by $\chi_{\mathrm{SC}}(\mathbf{q}=\mathbf{0})=\chi_{\mathrm{D}}(\mathbf{q}=(\pi, \pi))$.

Both finite next-nearest-neighbor hopping amplitudes and doping away from half filling break the above charge SU(2) symmetry of the Hamiltonian (1), lifting the degeneracy between the on-site $s$-wave spin-singlet pairing and charge density wave channel, as also demonstrated by the results of Sec. VI.
[1] S. Weinberg, The Quantum Theory of Fields (Cambridge University Press, Cambridge, 1995).
[2] K. G. Wilson, The renormalization group and critical phenomena, Rev. Mod. Phys. 55, 583 (1983). 
[3] F. J. Wegner and A. Houghton, Renormalization group equation for critical phenomena, Phys. Rev. A 8, 401 (1973).

[4] P. W. Anderson, A poor man's derivation of scaling laws for the Kondo problem, J. Phys. C 3, 2436 (1970).

[5] K. G. Wilson, The renormalization group: Critical phenomena and the Kondo problem, Rev. Mod. Phys. 47, 773 (1975).

[6] P. Mehta and D. J. Schwab, An exact mapping between the variational renormalization group and deep learning, arXiv:1410.3831.

[7] D. Zanchi and H. J. Schulz, Instabilities of weakly correlated electronic gas on a two dimensional lattice, Z. Phys. B 103, 339 (1996).

[8] D. Zanchi and H. J. Schulz, Weakly correlated electrons on a square lattice: A renormalization group theory, Europhys. Lett. 44, 235 (1998).

[9] C. J. Halboth and W. Metzner, $d$-Wave Superconductivity and Pomeranchuk Instability in the Two-Dimensional Hubbard Model, Phys. Rev. Lett. 85, 5162 (2000).

[10] S.-W. Tsai and J. B. Marston, $\kappa-(B E D T-T T F)_{2} X$ organic crystals: Superconducting versus antiferromagnetic instabilities in an anisotropic triangular lattice Hubbard model, Can. J. Phys. 79, 1463 (2001).

[11] C. Honerkamp, M. Salmhofer, N. Furukawa, and T. M. Rice, Breakdown of the Landau-Fermi liquid in two dimensions due to umklapp scattering, Phys. Rev. B 63, 035109 (2001).

[12] M. Salmhofer, Continuous renormalization for fermions and Fermi liquid theory, Commun. Math. Phys. 194, 249 (1998).

[13] J. Sólyom, The Fermi gas model of one-dimensional conductors, Adv. Phys. 28, 201 (1979).

[14] J. Polchinski, Renormalization and effective lagrangians, Nucl. Phys. B 231, 269 (1984).

[15] C. Wetterich, Exact evolution equation for the effective potential, Phys. Lett. B 301, 90 (1993).

[16] P. Kopietz, L. Bartosch, and F. Schütz, Introduction to the Functional Renormalization Group (Springer, Berlin, 2010).

[17] J. Polchinski, Effective field theory and the Fermi surface, arXiv:hep-th/9210046.

[18] R. Shankar, Renormalization-group approach to interacting fermions, Rev. Mod. Phys. 66, 129 (1994).

[19] Y. M. Vilk and A.-M. S. Tremblay, Non-perturbative manybody approach to the Hubbard model and single-particle pseudogap, J. Phys. (France) I 7, 1309 (1997).

[20] D. Bergeron, V. Hankevych, B. Kyung, and A. M. S. Tremblay, Optical and de conductivity of the two-dimensional Hubbard model in the pseudogap regime and across the antiferromagnetic quantum critical point including vertex corrections, Phys. Rev. B 84, 085128 (2011).

[21] G. Rohringer, A. Valli, and A. Toschi, Local electronic correlation at the two-particle level, Phys. Rev. B 86, 125114 (2012).

[22] H. Hafermann, Self-energy and vertex functions from hybridization-expansion continuous-time quantum Monte Carlo for impurity models with retarded interaction, Phys. Rev. B 89, 235128 (2014).

[23] V. Janiš and V. Pokorný, Critical metal-insulator transition and divergence in a two-particle irreducible vertex in disordered and interacting electron systems, Phys. Rev. B 90, 045143 (2014).

[24] J. Vučičević, J. Kokalj, R. Žitko, N. Wentzell, D. Tanasković, and J. Mravlje, Conductivity in the Square Lattice Hubbard Model at High Temperatures: Importance of Vertex Corrections, Phys. Rev. Lett. 123, 036601 (2019).
[25] R. Nourafkan, M. Côté, and A. M. S. Tremblay, Charge fluctuations in lightly hole-doped cuprates: Effect of vertex corrections, Phys. Rev. B 99, 035161 (2019).

[26] A. Kauch, P. Pudleiner, K. Astleithner, P. Thunström, T. Ribic, and K. Held, Generic Optical Excitations of Correlated Systems: $\pi$-tons, Phys. Rev. Lett. 124, 047401 (2020).

[27] D. Springer, P. Chalupa, S. Ciuchi, G. Sangiovanni, and A. Toschi, Interplay between local response and vertex divergences in many-fermion systems with on-site attraction, Phys. Rev. B 101, 155148 (2020).

[28] A. Toschi, R. Arita, P. Hansmann, G. Sangiovanni, and K. Held, Quantum dynamical screening of the local magnetic moment in Fe-based superconductors, Phys. Rev. B 86, 064411 (2012).

[29] M. Liu, L. W. Harriger, H. Luo, M. Want, R. A. Ewings, T. Guidi, H. Park, K. Haule, G. Kotliar, S. M. Hayden, and P. Dai, Nature of magnetic excitations in superconducting $\mathrm{BaFe}_{1.9} \mathrm{Ni}_{0.1} \mathrm{As}_{2}$, Nat. Phys. 8, 376 (2012).

[30] A. Galler, C. Taranto, M. Wallerberger, M. Kaltak, G. Kresse, G. Sangiovanni, A. Toschi, and K. Held, Screened moments and absence of ferromagnetism in FeAl, Phys. Rev. B 92, 205132 (2015).

[31] A. Hausoel, M. Karolak, E. Şaşı̆ı̆lu, A. Lichtenstein, K. Held, A. Katanin, A. Toschi, and G. Sangiovanni, Local magnetic moments in iron and nickel at ambient and earth's core conditions, Nat. Commun. 8, 16062 (2017).

[32] C. Watzenböck, M. Edelmann, D. Springer, G. Sangiovanni, and A. Toschi, Characteristic Time Scales of the Local Moment Dynamics in Hund's-Metals, Phys. Rev. Lett. 125, 086402 (2020).

[33] S. Andergassen, T. Enss, V. Meden, W. Metzner, U. Schollwöck, and K. Schönhammer, Functional renormalization group for Luttinger liquids with impurities, Phys. Rev. B 70, 075102 (2004).

[34] S. Andergassen, T. Enss, V. Meden, W. Metzner, U. Schollwöck, and K. Schönhammer, Renormalization-group analysis of the one-dimensional extended Hubbard model with a single impurity, Phys. Rev. B 73, 045125 (2006).

[35] A. A. Katanin and A. P. Kampf, Quasiparticle Anisotropy and Pseudogap Formation from the Weak-Coupling Renormalization Group Point of View, Phys. Rev. Lett. 93, 106406 (2004).

[36] D. Rohe and W. Metzner, Pseudogap at hot spots in the twodimensional Hubbard model at weak coupling, Phys. Rev. B 71, 115116 (2005).

[37] F. Wang, H. Zhai, Y. Ran, A. Vishwanath, and D.-H. Lee, Functional Renormalization-Group Study of the Pairing Symmetry and Pairing Mechanism of the FeAs-Based High-Temperature Superconductor, Phys. Rev. Lett. 102, 047005 (2009).

[38] C. Platt, W. Hanke, and R. Thomale, Functional renormalization group for multi-orbital Fermi surface instabilities, Adv. Phys. 62, 453 (2013).

[39] C. Honerkamp, Density Waves and Cooper Pairing on the Honeycomb Lattice, Phys. Rev. Lett. 100, 146404 (2008).

[40] M. L. Kiesel, C. Platt, W. Hanke, D. A. Abanin, and R. Thomale, Competing many-body instabilities and unconventional superconductivity in graphene, Phys. Rev. B 86, 020507(R) (2012).

[41] W.-S. Wang, Y.-Y. Xiang, Q.-H. Wang, F. Wang, F. Yang, and D.-H. Lee, Functional renormalization group and variational Monte Carlo studies of the electronic instabilities 
in graphene near $\frac{1}{4}$ doping, Phys. Rev. B 85, 035414 (2012).

[42] S. Raghu, X.-L. Qi, C. Honerkamp, and S.-C. Zhang, Topological Mott Insulators, Phys. Rev. Lett. 100, 156401 (2008).

[43] D. D. Scherer, M. M. Scherer, and C. Honerkamp, Correlated spinless fermions on the honeycomb lattice revisited, Phys. Rev. B 92, 155137 (2015).

[44] F. B. Kugler and J. von Delft, Multiloop Functional Renormalization Group that Sums up All Parquet Diagrams, Phys. Rev. Lett. 120, 057403 (2018).

[45] F. B. Kugler and J. von Delft, Multiloop functional renormalization group for general models, Phys. Rev. B 97, 035162 (2018).

[46] P. Chalupa, C. Hille, F. B. Kugler, J. von Delft, S. Andergassen, and A. Toschi (unpublished).

[47] C. Karrasch, R. Hedden, R. Peters, T. Pruschke, K. Schönhammer, and V. Meden, A finite-frequency functional renormalization group approach to the single impurity Anderson model, J. Phys.: Condens. Matter 20, 345205 (2008).

[48] C. Husemann and M. Salmhofer, Efficient parametrization of the vertex function, $\Omega$ scheme, and the $t, t^{\prime}$ Hubbard model at van Hove filling, Phys. Rev. B 79, 195125 (2009).

[49] K.-U. Giering and M. Salmhofer, Self-energy flows in the twodimensional repulsive Hubbard model, Phys. Rev. B 86, 245122 (2012).

[50] A. Eberlein, Self-energy effects in functional renormalization group flows of the two-dimensional $t-t^{\prime}$ Hubbard model away from van Hove filling, Phys. Rev. B 92, 235146 (2015).

[51] A. Eberlein, Fermionic two-loop functional renormalization group for correlated fermions: Method and application to the attractive Hubbard model, Phys. Rev. B 90, 115125 (2014).

[52] S. A. Maier, A. Eberlein, and C. Honerkamp, Functional renormalization group for commensurate antiferromagnets: Beyond the mean-field picture, Phys. Rev. B 90, 035140 (2014).

[53] J. Lichtenstein, D. Sánchez de la Peña, D. Rohe, E. Di Napoli, C. Honerkamp, and S. Maier, High-performance functional renormalization group calculations for interacting fermions, Comput. Phys. Commun. 213, 100 (2017).

[54] D. Rohe, Hierarchical parallelisation of functional renormalisation group calculations - hp-fRG, Comput. Phys. Commun. 207, 160 (2016)

[55] C. J. Eckhardt, G. A. H. Schober, J. Ehrlich, and C. Honerkamp, Truncated-unity parquet equations: Application to the repulsive Hubbard model, Phys. Rev. B 98, 075143 (2018).

[56] C. J. Eckhardt, C. Honerkamp, K. Held, and A. Kauch, Truncated unity parquet solver, Phys. Rev. B 101, 155104 (2020).

[57] A. Tagliavini, C. Hille, F. B. Kugler, S. Andergassen, A. Toschi, and C. Honerkamp, Multiloop functional renormalization group for the two-dimensional Hubbard model: Loop convergence of the response functions, SciPost Phys. 6, 009 (2019).

[58] D. Vilardi, C. Taranto, and W. Metzner, Nonseparable frequency dependence of the two-particle vertex in interacting fermion systems, Phys. Rev. B 96, 235110 (2017).

[59] D. Vilardi, C. Taranto, and W. Metzner, Antiferromagnetic and $d$-wave pairing correlations in the strongly interacting twodimensional Hubbard model from the functional renormalization group, Phys. Rev. B 99, 104501 (2019).

[60] J. Schwinger, On the Green's functions of quantized fields. I, Proc. Natl. Acad. Sci. USA 37, 452 (1951).

[61] F. J. Dyson, The $s$ matrix in quantum electrodynamics, Phys. Rev. 75, 1736 (1949).
[62] C. Taranto, S. Andergassen, J. Bauer, K. Held, A. Katanin, W. Metzner, G. Rohringer, and A. Toschi, From Infinite to Two Dimensions through the Functional Renormalization Group, Phys. Rev. Lett. 112, 196402 (2014).

[63] N. Wentzell, C. Taranto, A. Katanin, A. Toschi, and S. Andergassen, Correlated starting points for the functional renormalization group, Phys. Rev. B 91, 045120 (2015).

[64] W. Metzner, M. Salmhofer, C. Honerkamp, V. Meden, and K. Schönhammer, Functional renormalization group approach to correlated fermion systems, Rev. Mod. Phys. 84, 299 (2012).

[65] A. A. Katanin, Fulfillment of Ward identities in the functional renormalization group approach, Phys. Rev. B 70, 115109 (2004).

[66] M. Rück and J. Reuther, Effects of two-loop contributions in the pseudofermion functional renormalization group method for quantum spin systems, Phys. Rev. B 97, 144404 (2018).

[67] F. B. Kugler and J. von Delft, Derivation of exact flow equations from the self-consistent parquet relations, New J. Phys. 20, 123029 (2018).

[68] S. A. Maier, J. Ortloff, and C. Honerkamp, Multiorbital effects in the functional renormalization group: A weak-coupling study of the Emery model, Phys. Rev. B 88, 235112 (2013).

[69] N. Wentzell, G. Li, A. Tagliavini, C. Taranto, G. Rohringer, K. Held, A. Toschi, and S. Andergassen, High-frequency asymptotics of the vertex function: Diagrammatic parametrization and algorithmic implementation, Phys. Rev. B 102, 085106 (2020).

[70] C. Hille, The role of the self-energy in the functional renormalization group description of interacting Fermi systems, Ph.D. thesis, Universität Tübingen, 2020

[71] N. D. Mermin and H. Wagner, Absence of Ferromagnetism or Antiferromagnetism in One- or Two-Dimensional Isotropic Heisenberg Models, Phys. Rev. Lett. 17, 1133 (1966).

[72] N. E. Bickers, in Theoretical Methods for Strongly Correlated Electrons, edited by D. Sénéchal, A.-M. Tremblay, and C. Bourbonnais (Springer, New York, 2004), pp. 237-296.

[73] B. Roulet, J. Gavoret, and P. Nozières, Singularities in the $\mathrm{X}$-ray absorption and emission of metals. I. First-order parquet calculation, Phys. Rev. 178, 1072 (1969).

[74] K. Veschgini and M. Salmhofer, Schwinger-Dyson renormalization group, Phys. Rev. B 88, 155131 (2013).

[75] G. Li, N. Wentzell, P. Pudleiner, P. Thunström, and K. Held, Efficient implementation of the parquet equations: Role of the reducible vertex function and its kernel approximation, Phys. Rev. B 93, 165103 (2016).

[76] G. Li, A. Kauch, P. Pudleiner, and K. Held, The victory project v1.0: An efficient parquet equations solver, Comput. Phys. Commun. 241, 146 (2019).

[77] R. Blankenbecler, D. J. Scalapino, and R. L. Sugar, Monte Carlo calculations of coupled boson-fermion systems. I, Phys. Rev. D 24, 2278 (1981).

[78] D. J. Scalapino and R. L. Sugar, Monte Carlo calculations of coupled boson-fermion systems. II, Phys. Rev. B 24, 4295 (1981).

[79] F. Assaad and H. Evertz, in Computational Many-Particle Physics, edited by H. Fehske, R. Schneider, and A. Weiße, Lecture Notes in Physics Vol. 739 (Springer, Berlin, 2008), pp. 277-356.

[80] C.-C. Chang, S. Gogolenko, J. Perez, Z. Bai, and R. T. Scalettar, Recent advances in determinant quantum Monte Carlo, Philos. Mag. 95, 1260 (2015). 
[81] Y.-Y. He, M. Qin, H. Shi, Z.-Y. Lu, and S. Zhang, Finite-temperature auxiliary-field quantum Monte Carlo: Self-consistent constraint and systematic approach to low temperatures, Phys. Rev. B 99, 045108 (2019).

[82] Y.-Y. He, H. Shi, and S. Zhang, Reaching the Continuum Limit in Finite-Temperature $A b$ Initio Field-Theory Computations in Many-Fermion Systems, Phys. Rev. Lett. 123, 136402 (2019).

[83] L. Boehnke, H. Hafermann, M. Ferrero, F. Lechermann, and O. Parcollet, Orthogonal polynomial representation of imaginarytime Green's functions, Phys. Rev. B 84, 075145 (2011).

[84] G. Rohringer, A. Toschi, A. Katanin, and K. Held, Critical Properties of the Half-Filled Hubbard Model in Three Dimensions, Phys. Rev. Lett. 107, 256402 (2011).

[85] T. Schäfer, N. Wentzell, F. Šimkovic, Y.-Y. He, C. Hille, M. Klett, C. J. Eckhardt, B. Arzhang, V. Harkov, F.-M. Le Régent, A. Kirsch, Y. Wang, A. Kim, E. Kozik, E. A. Stepanov, A. Kauch, S. Andergassen, P. Hansmann, D. Rohe, Y. M. Vilk, J. LeBlanc, S. Zhang, A. M. Tremblay, M. Ferrero, O. Parcollet, and A. Georges, Tracking the footprints of spin fluctuations: A multi-method, multi-messenger study of the two-dimensional Hubbard model, arXiv:2006.10769.

[86] A. J. Kim, F. Simkovic, and E. Kozik, Spin and Charge Correlations across the Metal-to-Insulator Crossover in the Half-Filled 2D Hubbard Model, Phys. Rev. Lett. 124, 117602 (2020).

[87] T. Schäfer, F. Geles, D. Rost, G. Rohringer, E. Arrigoni, K. Held, N. Blümer, M. Aichhorn, and A. Toschi, Fate of the false Mott-Hubbard transition in two dimensions, Phys. Rev. B 91, 125109 (2015).
[88] T. Schäfer, A. Toschi, and K. Held, Dynamical vertex approximation for the two-dimensional hubbard model, J. Magn. Magn. Mater. 400, 107 (2016).

[89] F. Šimkovic IV, J. P. F. LeBlanc, A. J. Kim, Y. Deng, N. V. Prokof'ev, B. V. Svistunov, and E. Kozik, Extended Crossover from a Fermi Liquid to a Quasiantiferromagnet in the HalfFilled 2D Hubbard Model, Phys. Rev. Lett. 124, 017003 (2020).

[90] D. Vilardi, C. Taranto, and W. Metzner, Dynamically enhanced magnetic incommensurability: Effects of local dynamics on nonlocal spin correlations in a strongly correlated metal, Phys. Rev. B 97, 235110 (2018).

[91] A. A. Katanin, Two-loop functional renormalization group approach to the one- and two-dimensional Hubbard model, Phys. Rev. B 79, 235119 (2009).

[92] C. Hille, D. Rohe, C. Honerkamp, and S. Andergassen, Pseudogap opening in the two-dimensional Hubbard model: A functional renormalization group analysis, Phys. Rev. Res. 2, 033068 (2020).

[93] M. C. T. D. Müller, S. Blügel, and C. Friedrich, Electronmagnon scattering in elementary ferromagnets from first principles: Lifetime broadening and band anomalies, Phys. Rev. B 100, 045130 (2019).

[94] D. Krause and P. Thörnig, JURECA: Modular supercomputer at Jülich Supercomputing Centre, J. Large-Scale Res. facilities 4, A132 (2018).

[95] R. T. Scalettar, E. Y. Loh, J. E. Gubernatis, A. Moreo, S. R. White, D. J. Scalapino, R. L. Sugar, and E. Dagotto, Phase Diagram of the Two-Dimensional Negative- $U$ Hubbard Model, Phys. Rev. Lett. 62, 1407 (1989). 